KUCP0220

UTAP-422

October 3, 2002

\title{
Cosmology with Radion and Bulk Scalar Field in Two Branes Model
}

\author{
Shinpei Kobayashi ${ }^{1}$ and Kazuya Koyama ${ }^{2}$ \\ ${ }^{1}$ Graduate School of Human and Environment Studies, Kyoto University, Kyoto 606-8501, Japan \\ ${ }^{2}$ Department of Physics, The University of Tokyo, Tokyo 113-0033, Japan
}

\footnotetext{
${ }^{1}$ E-mail: shinpei@phys.h.kyoto-u.ac.jp

${ }^{2}$ E-mail: kazuya@utap.phys.s.u-tokyo.ac.jp
} 


\begin{abstract}
We investigate cosmological evolutions of the bulk scalar field $\phi(t)$ and the radion $d(t)$ in five-dimensional dilatonic two branes model. The bulk potential for the scalar field is taken as the exponential function $V_{b u l k} \propto \exp (-2 \sqrt{2} b \phi)$, where $b$ is the parameter of the theory. This model includes Randall-Sundrum model (with $b=0$ ) and fivedimensional Horava-Witten theory (with $b=1$ ). We consider matter on both branes and arbitrary potentials on the branes and in the bulk. These matter and potentials induce the cosmological expansion of the brane as well as the time evolution of the bulk scalar field and the radion. Starting with full five-dimensional equations, we derive four-dimensional effective equations which govern the low-energy dynamics of brane worlds. A correspondent five-dimensional geometry is also obtained. The effective fourdimensional theory on a positive tension brane is described by bi-scalar tensor theory. If the radion is stabilized, the effective theory becomes Brans-Dicke (BD) theory with BD parameter $1 / 2 b^{2}$. On the other hand, if the scalar field is stabilized, the effective theory becomes scalar-tensor theory with BD parameter $\frac{3}{2\left(3 b^{2}+1\right)} \frac{\varphi(t)}{1-\varphi(t)}$ where $\varphi$ is the BD field defined by radion $d(t)$. If we do not introduce the stabilization mechanism for these moduli fields, the acceptable late time cosmology can be realized only if the dilaton coupling $b$ is small $\left(b^{2}<1.6 \times 10^{-4}\right)$ and the negative tension brane is sufficiently away from the positive tension brane. We also construct several models for inflationary brane worlds driven by potentials on the brane and in the bulk.
\end{abstract}




\section{Introduction}

String Theory and/or M-Theory are thought to be the most promising candidate for "The Theory of Everything". One of the interesting and embarrassing features of such theories is that they are consistently formulated in more than four-dimensions. On the other hand, at least at low energy scales, our spacetime seems to be four-dimensional. Hence we need some compactification mechanisms of extra dimensions in order to retrieve our four-dimensional spacetime at low energy scales.

Of all compactification mechanisms of the extra dimensions, Hořava-Witten (HW) theory is a fascinating one 1] 2]. Hořava and Witten showed that the eleven-dimensional limit of M-theory compactified on $S^{1} / \mathbf{Z}_{2}$ orbifold can be identified as the strongly coupled ten-dimensional $E_{8} \times E_{8}$ heterotic string theory with two orbifold fixed planes. Witten also showed that there exists a consistent compactification of this theory where the universe appears as a five-dimensional spacetime with two boundary branes [3]. Then the five dimensional effective action for this theory was derived [4] and the cosmological evolution of the branes has been studied [5]-[8]. ${ }^{3}$ In this model, the bulk scalar field is necessarily associated with the compactification.

The bulk scalar field also plays an important role in phenomenological models for brane worlds. For example, the bulk inflaton models [9]-12], the self-tuning models for cosmological constant [13]-17] and the dilatonic thick domain wall models [18] - 21] have been studied as the extension of Randall-Sundrum (RS) models [22], 23]. Cosmological models with bulk scalars have been actively considered [24, 25]. In the context of RS model, stabilization mechanism of the distance between two branes using bulk scalars has been investigated [26]-33].

As well as the bulk scalar field, there is another important scalar degree of freedom in two branes

\footnotetext{
${ }^{3}$ Note that the time evolution of their cosmological domain wall solution is driven by the bulk scalar field, not by the matter on the brane. So their solution is a "vacuum cosmological solution", which is different from the cosmological solution in our usual sense.
} 
model, that is, a radion which describes the inter-brane distance. The radion plays a crucial role in gravitational interactions on the brane. For example, in RS model, the radion acts as a Brans-Dicke (BD) field and the observational constraints on BD parameter gives strong constraint on the model. In RS model, the dynamics of the radion has been eagerly studied [34-40].

The purpose of this paper is to investigate the cosmological consequences of the bulk scalar field and the radion. We consider a model where the bulk potential for the bulk scalar field $\phi$ is given by an exponential function $V_{b u l k} \propto \exp (-2 \sqrt{2} b \phi)$. This model includes HW theory (with $b=1$ ) and RS model (with $b=0$ ). We will consider matter on both branes and arbitrary potentials on the branes and in the bulk. These matter and potentials induce the cosmological expansion of the brane as well as the time evolution of the bulk scalar field and the radion. It is well known that in many higher-dimensional theories, these "moduli fields" are potentially dangerous because time evolution of moduli fields leads to the time variation of fundamental constants. Then it is important to know how serious is the moduli problem in this model. We also consider the phenomenological implication of the potentials mainly focusing on the inflationary scenario.

In order to know the cosmological consequences of the bulk scalar field and radion, we should derive the effective equations which govern the cosmological dynamics of these fields. One approach is to construct an $4 \mathrm{D}$ Effective action by the integration of the action with respect to the extra dimension [41-44]. A "moduli space" approximation is an example for such an approach. In this approximation, the constant moduli parameters of the static solution is replaced by time-dependent functions and integration over the extra dimensions gives four-dimensional effective action. But, it is known that the moduli space approximation may not always be consistent [45]- [47] and the integration over extra-dimension must be treated with special caution [48].

Hence, in this paper, we will derive four-dimensional effective equations for the radion and the bulk scalar field starting with full five-dimensional equations. In order to do so, we use the lowenergy expansion scheme. The low-energy means that the energy density of the matter on the 
brane is much lower than the tension of the brane. This relation holds at late times in cosmological evolution. Under such an approximation, we can derive the effective four-dimensional equations for scale factor, radion and bulk scalar field. We can also determine the correspondent five-dimensional geometry.

The organization of this paper as follows. In Sec. II, we review the setup of our model and introduce the low-energy approximation. Using this approximation, we give the background solutions. In Sec. III, we solve the first-order equations and derive the solutions for full five-dimensional metric at this order. Using these solutions, we show that the equations for scale factor on the brane, bulk scalar and radion can be derived. Sec. IV is the main part of this paper. Effective equations which govern the low energy dynamics of the two branes system are presented. The comparison with scalar-tensor theory is performed and the observational constraints are obtained. Sec. V, several inflationary scenarios are discussed. Sec. VI is devoted to conclusions.

\section{Setup}

\subsection{Action and Equations of Motion}

We consider the bulk action of the form

$$
S_{b u l k}=\frac{1}{2 \kappa^{2}} \int_{M_{5}} d^{5} x \sqrt{-g_{5}}\left(R-\partial_{M} \phi \partial^{M} \phi+V_{b u l k}(\phi)\right)
$$

where $\kappa^{2}$ denotes the five-dimensional gravitational constant and $\sigma$ denotes the tension of the 3brane. The bulk potential is taken as

$$
V_{b u l k}(\phi)=-\left(b^{2}-\frac{2}{3}\right) e^{-2 \sqrt{2} b \phi} \sigma^{2}+V(\phi) .
$$

Here we split $V_{b u l k}$ into an explicit, exponential potential for $\phi$ and an arbitrary potential $V(\phi)$ according to Ref. 49]. The parameter $b$ is determined by the theory. If we choose $b=0$ and $V(\phi)=0$, we retrieve the action for the Randall-Sundrum model and if $b=1$ and $V(\phi)=0$, we retrieve the action for Hořava-Witten model, which were derived in [4]. 
We consider a five-dimensional spacetime with structure $M_{5}=S^{1} / \mathbf{Z}_{2} \times M_{4}$ where $S^{1} / \mathbf{Z}_{2}$ is an orbifold and $M_{4}$ is a smooth manifold. We use the coordinate $y$ to parameterize the orbifold and the range of $y$ is chosen as $-r \leq y \leq r$. Because this spacetime has $\mathbf{Z}_{2}$-symmetry (i.e. we identify $y$ with $-y$ ), there are two endpoints in this spacetime. These two endpoints are called orbifold fixed planes, which are 3-branes. A positive tension brane is set at $y=0$ and a negative tension brane is set at $y=r$. We take the actions for them as

$$
\begin{aligned}
S_{\text {brane }} & =S_{+}+S_{-} \\
S_{+} & =\int_{M_{4}^{(1)}} d^{4} x \sqrt{-g_{4}} U_{\text {brane }}+\int_{M_{4}^{(1)}} d^{4} x \sqrt{-g_{4}} \mathcal{L}_{m} \\
S_{-} & =\int_{M_{4}^{(2)}} d^{4} x \sqrt{-g_{4}} \tilde{U}_{\text {brane }}+\int_{M_{4}^{(2)}} d^{4} x \sqrt{-g_{4}} \tilde{\mathcal{L}}_{m}
\end{aligned}
$$

where the potentials are given by

$$
\begin{aligned}
& U_{\text {brane }}=-\frac{\sqrt{2}}{\kappa^{2}} e^{-\sqrt{2} b \phi} \sigma-U(\phi), \\
& \tilde{U}_{\text {brane }}=\frac{\sqrt{2}}{\kappa^{2}} e^{-\sqrt{2} b \phi} \sigma-\tilde{U}(\phi) .
\end{aligned}
$$

The action for the positive tension brane and the negative tension brane is denoted by $S_{+}$and $S_{-}$, respectively. $U(\phi)$ and $\tilde{U}(\phi)$ are arbitrary potentials. $\mathcal{L}_{m}$ and $\tilde{\mathcal{L}}_{m}$ are the Lagrangian density of the matter on each brane. We assume that they do not couple to the bulk scalar, that is, $\delta \mathcal{L}_{m} / \delta \phi=\delta \tilde{\mathcal{L}}_{m} / \delta \phi=0$. It is true that in HW model, there are field strengths of $E_{8}$ and $E_{6}$ gauge field which couple to the bulk scalar field, but in this paper, we replace them with the energy density of perfect fluids ${ }^{4}$.

Using the action, we derive the Einstein equation

$$
G_{N}^{M}=\kappa^{2}\left(T_{\text {bulk } N}^{M}+T_{\text {brane } N}^{M}\right) .
$$

\footnotetext{
${ }^{4}$ It is also interesting to consider the coupling of the gauge field and the scalar field. It is left for the future work.
} 
The energy-momentum tensor can be divided into two parts;

$$
\begin{aligned}
T_{\text {bulk } N}^{M}= & \frac{1}{\kappa^{2}}\left\{\nabla^{M} \phi \nabla_{N} \phi-\frac{1}{2} \delta_{N}^{M}\left[\nabla^{M} \phi \nabla_{M} \phi+\left(b^{2}-\frac{2}{3}\right) e^{-2 \sqrt{2} b \phi} \sigma^{2}-V(\phi)\right]\right\}, \\
T_{\text {brane } N}^{M}= & \frac{\sqrt{-g_{4}}}{\sqrt{-g_{5}}}\left\{\left[-\frac{\sqrt{2}}{\kappa^{2}} e^{-\sqrt{2} b \phi} \operatorname{diag}(0, \sigma, \sigma, \sigma, \sigma)+\operatorname{diag}(0,-\rho-U, p-U, p-U, p-U)\right] \delta(y)\right. \\
& \left.+\left[\frac{\sqrt{2}}{\kappa^{2}} e^{-\sqrt{2} b \phi} \operatorname{diag}(0, \sigma, \sigma, \sigma, \sigma)+\operatorname{diag}(0,-\tilde{\rho}-\tilde{U}, \tilde{p}-\tilde{U}, \tilde{p}-\tilde{U}, \tilde{p}-\tilde{U})\right] \delta(y-r)\right\}(6)
\end{aligned}
$$

The energy density and the pressure of the perfect fluid on the positive tension brane are denoted by $\rho$ and $p$ and those on the negative tension brane are denoted by $\tilde{\rho}$ and $\tilde{p}$. The equation of motion for the scalar field is given by

$$
\begin{aligned}
\nabla^{2} \phi+\sqrt{2} b\left(b^{2}-\frac{2}{3}\right) & e^{-2 \sqrt{2} b \phi} \sigma^{2}+\frac{1}{2} \frac{d V}{d \phi} \\
& =\frac{\sqrt{-g_{4}}}{\sqrt{-g_{5}}}\left[\left(-2 b e^{-\sqrt{2} b \phi} \sigma+\kappa^{2} \frac{d U}{d \phi}\right) \delta(y)+\left(2 b e^{-\sqrt{2} b \phi} \sigma+\kappa^{2} \frac{d \tilde{U}}{d \phi}\right) \delta(y-r)\right]
\end{aligned}
$$

Now we take the five-dimensional metric as

$$
d s^{2}=e^{2 \gamma(y, t)} d y^{2}-e^{2 \beta(y, t)} d t^{2}+e^{2 \alpha(y, t)} \delta_{i j} d x^{i} d x^{j}
$$

Einstein equations and the scalar field equation in terms of $\alpha, \beta, \gamma$ and $\phi$ are presented in Appendix A-1. We expand the functions around $y=0$ and $y=r$ as

$$
\begin{aligned}
& \alpha(y, t)=\alpha^{(0)}(t)+\alpha^{(1)}(t)|y|+\frac{1}{2} \alpha^{(2)}(t) y^{2}+\cdots \\
& \alpha(y, t)=\tilde{\alpha}^{(0)}(t)+\tilde{\alpha}^{(1)}(t)|r-y|+\frac{1}{2} \tilde{\alpha}^{(2)}(t)(r-y)^{2}+\cdots
\end{aligned}
$$

From above expansions, we can read that $\alpha^{\prime \prime}$ gives $2 \delta(y)-2 \delta(y-r)$. Then from Einstein equations $\alpha^{(1)}$ and $\tilde{\alpha}^{(1)}$ can be written in terms of $\rho, p, U$ and $\tilde{\rho}, \tilde{p}, \tilde{U}$ respectively. Then we can get the 
junction conditions;

$$
\begin{aligned}
& \alpha^{(1)}(t)=-\left.\frac{1}{6}\left[\sqrt{2} \sigma e^{-\sqrt{2} b \phi}+\kappa^{2}(\rho+U)\right] e^{\gamma}\right|_{y=0}, \\
& \beta^{(1)}(t)=-\left.\frac{1}{6}\left[\sqrt{2} \sigma e^{-\sqrt{2} b \phi}-\kappa^{2}(3 p+2 \rho-U)\right] e^{\gamma}\right|_{y=0}, \\
& \phi^{(1)}(t)=-\left.\left[b \sigma e^{-\sqrt{2} b \phi}-\frac{1}{2} \kappa^{2} \frac{d U}{d \phi}\right] e^{\gamma}\right|_{y=0}, \\
& \tilde{\alpha}^{(1)}(t)=-\left.\frac{1}{6}\left[\sqrt{2} \sigma e^{-\sqrt{2} b \phi}-\kappa^{2}(\tilde{\rho}+\tilde{U})\right] e^{\gamma}\right|_{y=r}, \\
& \tilde{\beta}^{(1)}(t)=-\left.\frac{1}{6}\left[\sqrt{2} \sigma e^{-\sqrt{2} b \phi}+\kappa^{2}(3 \tilde{p}+2 \tilde{\rho}-\tilde{U})\right] e^{\gamma}\right|_{y=r}, \\
& \tilde{\phi}^{(1)}(t)=-\left.\left[b \sigma e^{-\sqrt{2} b \phi}+\frac{1}{2} \kappa^{2} \frac{d \tilde{U}}{d \phi}\right] e^{\gamma}\right|_{y=r} .
\end{aligned}
$$

\subsection{Low Energy Expansion Scheme}

In this subsection, we formulate the low energy expansion scheme to solve the equations ${ }^{5}$. At late times, the energy density and the pressure of the perfect fluid is much smaller than the tension of the brane. Hence we can impose the following conditions,

$$
\sigma \gg \kappa^{2} \rho, \kappa^{2} p, \sigma^{-1} V, \kappa^{2} U, \kappa^{2} \tilde{U}
$$

Then, we can define a small parameter of the system as follows,

$$
\varepsilon \equiv \frac{\kappa^{2} \rho}{\sigma}
$$

Thus for $\varepsilon \ll 1$, we can solve five-dimensional Einstein equations perturbatively by expanding a metric and scalar field as

$$
\alpha(y, t)=\alpha_{(0)}(y, t)+\alpha_{(1)}(y, t)+\alpha_{(2)}(y, t)+\cdots,
$$

where $\alpha_{(i)}$ is the function of the order $\mathcal{O}\left(\varepsilon^{i}\right)$. Note that the lower indices $\alpha_{(i)}$ are different from the upper indices $\alpha^{(i)}$ which have been defined in eqs.(8), (9). We expand $\beta(y, t), \gamma(y, t)$, and $\phi(y, t)$

\footnotetext{
${ }^{5}$ Generalizations to covariant form of this iteration scheme are performed in [50, [51]
} 
in a similar way. The important point is that $t$-derivative of the lower order solution is the same order as $y$-derivative of the higher order solution;

$$
\ddot{\alpha}_{(i-1)} \sim \alpha_{(i)}^{\prime \prime}
$$

This is because the derivative with respect to $y$ is of the order $\alpha^{\prime} \sim \sigma$ for $\varepsilon \ll 1$ from the junction conditions. On the other hand, from the Friedmann equation, we expect that the derivative with respect to $t$ is of the order $\dot{\alpha}^{2} \sim \kappa^{2} \sigma \rho$. Then the derivative with respect to $t$ is suppressed by the factor $\varepsilon^{1 / 2}$ compared with the derivative with respect to $y$;

$$
\left(\frac{\dot{\alpha}}{\alpha^{\prime}}\right)^{2} \sim \varepsilon .
$$

Hence, the leading order equation of the order $\mathcal{O}\left(\epsilon^{0}\right)$ contains only the $y$-derivative of the 0-th order solution (i.e., $\left.\alpha_{(0)}^{\prime \prime}, \beta_{(0)}^{\prime \prime}, \cdots\right)$, and 1st order equations are consisted of $t$-derivatives of 0 -th order functions and $y$-derivatives of 1 st order functions (i.e., $\ddot{\alpha}_{(0)}, \ddot{\beta}_{(0)}, \cdots, \alpha_{(1)}^{\prime \prime}, \beta_{(1)}^{\prime \prime}, \cdots$ ) as shown in Appendix A. At each order, Einstein equations give ordinary differential equations for $\left(\alpha_{(i)}, \beta_{(i)}, \cdots\right)$ with respect to $y$. Then it is possible to solve the equations analytically.

\subsection{Solution at Zeroth Order}

The concrete form of the 0 -th order equations are given in Appendix A-2 and the solutions at the 0-th order are given as follows,

$$
\begin{aligned}
\alpha_{(0)}(y, t) & =\frac{2}{3 \Delta+8} \ln H(y, t)+\hat{\alpha}(t), \\
\beta_{(0)}(y, t) & =\frac{2}{3 \Delta+8} \ln H(y, t) \\
\gamma_{(0)}(y, t) & =\ln d(t)+\sqrt{2} b \hat{\phi}(t) \\
\phi_{(0)}(y, t) & =\frac{1}{\sqrt{2} b} \ln H(y, t)+\hat{\phi}(t)
\end{aligned}
$$

where,

$$
H(y, t) \equiv 1-\frac{\sqrt{2}(3 \Delta+8)}{12} d(t) \sigma|y|,
$$


and

$$
\Delta \equiv 4\left(b^{2}-\frac{2}{3}\right)
$$

Here $\hat{\alpha}(t)$ can be identified with the scale factor on a positive tension brane and $\hat{\phi}(t)$ describes the behavior of the scalar field. $d(t)$ describes the time evolution of inter-brane distance. There does not exist $\hat{\beta}(t)$ because $\beta_{(0)}$ corresponds to the lapse function, so we can always gauge away $\hat{\beta}(t)$ by the redefinition of the time variable $t$. These zeroth-order solutions are quasi-static and their time evolutions will be determined by the first-order solutions. If we take $\hat{\alpha}(t)=\hat{\phi}(t)=0$ and $d(t)=1$, we can retrieve the vacuum solution obtained in [52]- 54$]$.

The above solutions are valid except for RS model with $b=0(\Delta=-8 / 3)$. For RS model, the 0-th order solutions are given by

$$
\begin{aligned}
\alpha_{(0)}(y, t) & =-d(t) k|y|+\hat{\alpha}(t), \\
\beta_{(0)}(y, t) & =-d(t) k|y|, \\
\gamma_{(0)}(y, t) & =\ln d(t), \\
\phi_{(0)}(y, t) & =\hat{\phi}(t),
\end{aligned}
$$

where $k=\sqrt{2} \sigma / 6$ is the curvature scale of the bulk Anti- de Sitter spacetime. In the following, we assume $b \neq 0$ and RS model is treated separately in Appendix C. 


\section{Solutions for bulk metric and scalar field}

\subsection{Solutions at First Order}

\subsubsection{Equations}

The concrete form of the 1-th order equations are given in Appendix A-3. Substituting the solutions at 0 -th order into the equations at 1 st order, we get

$$
\begin{aligned}
& -\frac{\sqrt{2}}{6} \frac{\sigma d}{H}\left(3 \alpha_{(1)}^{\prime}+\beta_{(1)}^{\prime}-\sqrt{2} b \phi_{(1)}^{\prime}\right)+\frac{1}{3}\left(b^{2}-\frac{2}{3}\right) \frac{\sigma^{2} d^{2}}{H^{2}}\left(\gamma_{(1)}-\sqrt{2} b \phi_{(1)}\right) \\
& =d^{2} e^{2 \sqrt{2} b \hat{\phi}}\left\{H ^ { - \frac { 1 } { 3 b ^ { 2 } } } \left[\ddot{\hat{\alpha}}+2 \dot{\hat{\alpha}}^{2}+\frac{1}{6} \dot{\hat{\phi}}^{2}-\frac{\sqrt{2}}{6} \frac{\sigma}{H} y(\ddot{d}+3 \dot{d} \dot{\hat{\alpha}}+\sqrt{2} b \dot{d} \dot{\hat{\phi}})\right.\right. \\
& \left.\left.+\frac{1-3 b^{2}}{18} \frac{\sigma^{2}}{H^{2}} \dot{d}^{2} y^{2}\right]+\frac{1}{6} V\right\} \\
& \alpha_{(1)}^{\prime \prime}-\frac{\sqrt{2}}{6} \frac{\sigma d}{H}\left(4 \alpha_{(1)}^{\prime}-\gamma_{(1)}^{\prime}+\sqrt{2} b \phi_{(1)}^{\prime}\right)+\frac{1}{3}\left(b^{2}-\frac{2}{3}\right) \frac{\sigma^{2} d^{2}}{H^{2}}\left(\gamma_{(1)}-\sqrt{2} b \phi_{(1)}\right) \\
& =d^{2} e^{2 \sqrt{2} b \hat{\phi}}\left\{H ^ { - \frac { 1 } { 3 b ^ { 2 } } } \left[\dot{\hat{\alpha}}^{2}+\sqrt{2} \dot{\hat{\alpha}} \dot{\hat{\phi}}-\frac{1}{6} \dot{\hat{\phi}}^{2}+\frac{\dot{d}}{d} \dot{\hat{\alpha}}-\frac{\sqrt{2}}{6} \frac{\sigma}{H} y\left(\frac{\dot{d}^{2}}{d}+2 \dot{d} \dot{\hat{\alpha}}\right)\right.\right. \\
& \left.\left.+\frac{1-3 b^{2}}{18} \frac{\sigma^{2}}{H^{2}} \dot{d}^{2} y^{2}\right]+\frac{1}{6} V\right\} \\
& 2 \alpha_{(1)}^{\prime \prime}+\beta_{(1)}^{\prime \prime}-\frac{\sqrt{2}}{6} \frac{\sigma d}{H}\left(8 \alpha_{(1)}^{\prime}+4 \beta_{(1)}^{\prime}-3 \gamma_{(1)}^{\prime}+3 \sqrt{2} b \phi_{(1)}^{\prime}\right) \\
& +\left(b^{2}-\frac{2}{3}\right) \frac{\sigma^{2} d^{2}}{H^{2}}\left(\gamma_{(1)}-\sqrt{2} b \phi_{(1)}\right) \\
& =d^{2} e^{2 \sqrt{2} b \hat{\phi}}\left\{H ^ { - \frac { 1 } { 3 b ^ { 2 } } } \left[2 \ddot{\hat{\alpha}}+2 \sqrt{2} b \dot{\hat{\alpha}} \dot{\hat{\phi}}+3 \dot{\hat{\alpha}}^{2}+\sqrt{2} b \ddot{\hat{\phi}}+\left(2 b^{2}+\frac{1}{2}\right) \dot{\hat{\phi}}^{2}+2 \frac{\dot{d}}{d} \dot{\hat{\alpha}}+\frac{\ddot{d}}{d}+2 \sqrt{2} b \dot{\hat{\phi}} \frac{\dot{d}}{d}\right.\right. \\
& -\frac{\sqrt{2}}{6} \frac{\sigma}{H} y\left(2 \ddot{d}+4 \sqrt{2} b \dot{d} \dot{\hat{\phi}}+4 \dot{d} \dot{\hat{\alpha}}+\frac{\dot{d}^{2}}{d}\right) \\
& \left.\left.+\frac{1-3 b^{2}}{18} \frac{\sigma^{2}}{H^{2}} \dot{d}^{2} y^{2}\right]+\frac{1}{2} V\right\},
\end{aligned}
$$




$$
\begin{aligned}
& \alpha_{(1)}^{\prime}\left(\sqrt{2} b \dot{\hat{\phi}}+\frac{\dot{d}}{d}\right)-\frac{1}{3} \phi_{(1)}^{\prime}\left(-b \frac{\sigma}{H} \dot{d} y+\dot{\hat{\phi}}\right) \\
& =\dot{\alpha}_{(1)}^{\prime}+\frac{\sqrt{2}}{6} \frac{\sigma d}{H}\left(\dot{\gamma}_{(1)}-\sqrt{2} b \dot{\phi}_{(1)}\right)-\left(-\frac{\sqrt{2}}{6} \frac{\sigma}{H} \dot{d} y+\dot{\hat{\alpha}}\right)\left(\beta_{(1)}^{\prime}-\alpha_{(1)}^{\prime}\right), \\
& \phi_{(1)}^{\prime \prime}-\frac{\sigma d}{H}\left[b\left(3 \alpha_{(1)}^{\prime}+\beta_{(1)}^{\prime}-\gamma_{(1)}^{\prime}\right)+\frac{2 \sqrt{2}}{3} \phi_{(1)}^{\prime}\right]+2 \sqrt{2} b\left(b^{2}-\frac{2}{3}\right) \frac{\sigma^{2} d^{2}}{H^{2}}\left(\gamma_{(1)}-\sqrt{2} b \phi_{(1)}\right) \\
& =d^{2} e^{2 \sqrt{2} b \hat{\phi}}\left(H ^ { - \frac { 1 } { 3 b ^ { 2 } } } \left\{\ddot{\hat{\phi}}+3 \dot{\hat{\alpha}} \dot{\hat{\phi}}+\sqrt{2} b \dot{\hat{\phi}}^{2}+\frac{\dot{d}}{\dot{\phi}} \dot{\hat{\phi}}-\frac{\sigma}{H} y\left[b\left(\ddot{d}+\sqrt{2} \dot{d} \dot{\hat{\phi}}+3 \dot{d} \dot{\hat{\alpha}}+\frac{\dot{d}^{2}}{d}\right)+\frac{\sqrt{2}}{3} \dot{\hat{\phi} \dot{d}}\right]\right.\right. \\
& \left.\left.+\frac{\sqrt{2}}{3} b\left(1-3 b^{2}\right) \frac{\sigma^{2}}{H^{2}} \dot{d}^{2} y^{2}\right\}-\frac{1}{2} \frac{d V}{d \phi}\right) \text {. }
\end{aligned}
$$

These equations can be regarded as the ordinary differential equations for $\left(\alpha_{(1)}, \beta_{(1)}, \gamma_{(1)}, \phi_{(1)}\right)$ where the right-hand sides of the equations act as sources. Then the solutions are given by homogeneous solutions and particular solutions which are determined by $\dot{\hat{\alpha}}, \dot{\hat{\phi}}, \dot{d}$ and $V\left(\phi_{(0)}\right)$.

\subsubsection{Homogeneous Solutions}

The homogeneous solutions are given by

$$
\begin{aligned}
& \alpha_{(1)}=K_{\alpha}(t) H^{\frac{b^{2}-\frac{2}{3}}{b^{2}}}+F_{\alpha}(t)+P(y, t), \\
& \beta_{(1)}=K_{\beta}(t) H^{\frac{b^{2}-\frac{2}{3}}{b^{2}}}+F_{\beta}(t)+P(y, t), \\
& \gamma_{(1)}=K_{\gamma}(t) H^{\frac{b^{2}-\frac{2}{3}}{b^{2}}}+\sqrt{2} b F_{\phi}(t)+6 b^{2} P(y, t),-3 \sqrt{2} \frac{H}{\sigma d} P^{\prime}(y, t), \\
& \phi_{(1)}=K_{\phi}(t) H^{\frac{b^{2}-\frac{2}{3}}{b^{2}}}+F_{\phi}(t)+3 \sqrt{2} b P(y, t) .
\end{aligned}
$$

Here, $F_{\alpha}, F_{\beta}, F_{\phi}$ and $P$ are arbitrary functions and they do not affect the resultant effective

equations. On the other hand, $K_{\alpha}, K_{\beta}, K_{\gamma}, K_{\phi}$ carry the information of the bulk. These functions satisfy two constraints;

$$
\begin{aligned}
3 K_{\alpha}+K_{\beta}+K_{\gamma}-2 \sqrt{2} b K_{\phi} & =0 \\
\dot{K}_{\alpha}+\left(K_{\alpha}-K_{\beta}\right) \dot{\hat{\alpha}}-\left(\sqrt{2} b K_{\alpha}-\frac{1}{3} K_{\phi}\right) \dot{\hat{\phi}} & =\frac{2}{3 \Delta}\left(\dot{K}_{\gamma}-\sqrt{2} b \dot{K}_{\phi}\right) .
\end{aligned}
$$


These constraints are insufficient to determine the behavior of the homogeneous solutions. The homogeneous solutions will be determined by the junctions conditions at the branes.

We should note that in the case of RS model $(b=0)$ without scalar field $K_{\phi}=0$, these constraints are sufficient to obtain the concrete form of the homogeneous solutions. We obtain the equation for $K$ as

$$
\left(K_{\alpha}+\frac{1}{4} K_{\gamma}\right)^{\cdot}+4 \dot{\hat{\alpha}}\left(K_{\alpha}+\frac{1}{4} K_{\gamma}\right)=0
$$

Thus we find

$$
K_{\alpha}+\frac{1}{4} K_{\gamma}=\frac{1}{8} C e^{-4 \hat{\alpha}}
$$

where $C$ is the integration constant. It is well known that $C$ is related to the mass of the Black hole in the bulk and this term will induce "dark radiation" on the brane.

\subsubsection{Particular Solutions}

From eqs.(31)-(35), the particular solutions is given by

$$
\begin{aligned}
\alpha_{(1)} & =f_{\alpha}(t) H^{2-\left(1 / 3 b^{2}\right)}(y, t)+g_{\alpha}(t) H^{1-\left(1 / 3 b^{2}\right)}(y, t) y+h_{\alpha}(t) H^{-1 / 3 b^{2}}(y, t) y^{2}+k_{\alpha}(y, t) \\
\beta_{(1)} & =f_{\beta}(t) H^{2-\left(1 / 3 b^{2}\right)}(y, t)+g_{\beta}(t) H^{1-\left(1 / 3 b^{2}\right)}(y, t) y+h_{\beta}(t) H^{-1 / 3 b^{2}}(y, t) y^{2}+k_{\beta}(y, t) \\
\gamma_{(1)} & =f_{\gamma}(t) H^{2-\left(1 / 3 b^{2}\right)}(y, t)+g_{\gamma}(t) H^{1-\left(1 / 3 b^{2}\right)}(y, t) y+h_{\gamma}(t) H^{-1 / 3 b^{2}}(y, t) y^{2}+\sqrt{2} b k_{\phi}(y, t), \\
\phi_{(1)} & =f_{\phi}(t) H^{2-\left(1 / 3 b^{2}\right)}(y, t)+g_{\phi}(t) H^{1-\left(1 / 3 b^{2}\right)}(y, t) y+h_{\phi}(t) H^{-1 / 3 b^{2}}(y, t) y^{2}+k_{\phi}(y, t)
\end{aligned}
$$

The functions $\left(f_{\alpha}, g_{\alpha}, h_{\alpha}, f_{\beta}, g_{\beta}, h_{\beta}, f_{\gamma}, g_{\gamma}, h_{\gamma}, f_{\phi}, g_{\phi}, h_{\phi}\right)$ are determined by $\hat{\alpha}(t), \hat{\phi}(t)$ and $d(t)$. The solutions are presented in Appendix B. On the other hand, the solution $k(y, t)$ is determined 
by bulk potential $V$ as

$$
\begin{aligned}
k_{\alpha}^{\prime}(y, t)= & \frac{1}{6} d^{2} e^{2 \sqrt{2} b \hat{\phi}} H^{-2 / 3 b^{2}} \int_{0}^{y} d y H^{2 / 3 b^{2}} V \\
k_{\beta}^{\prime}(y, t)= & -\frac{1}{2} d^{2} e^{2 \sqrt{2} b \hat{\phi}} H^{-2 / 3 b^{2}} \int_{0}^{y} d y H^{2 / 3 b^{2}}\left(\sqrt{2} b \frac{d V}{d \phi}+\left(1+2 b^{2}\right) V\right) \\
& -\frac{\sqrt{2}}{2} \sigma^{-1} d e^{2 \sqrt{2} b \hat{\phi}} H V \\
k_{\phi}^{\prime}(y, t)= & -\frac{1}{2} d^{2} e^{2 \sqrt{2} b \hat{\phi}} H^{-2 / 3 b^{2}} \int_{0}^{y} d y H^{2 / 3 b^{2}}\left(\frac{d V}{d \phi}+\sqrt{2} b V\right) .
\end{aligned}
$$

\subsection{Derivation of effective Friedmann equation}

Here we will demonstrate how we can derive the Friedmann equation using the above solutions. We use the solutions which we have derived in the previous subsection and the junction conditions given in Appendix $\mathrm{A}$.

Projecting $\alpha_{(1)}^{\prime}$ onto $y=0$, we get

$$
\alpha_{(1)}^{\prime}(0, t)=\alpha_{(1)}^{(1)}=\frac{\sqrt{2}}{3} \sigma d\left(1-6 b^{2}\right) f_{\alpha}(t)+g_{\alpha}(t)+k_{\alpha}^{\prime}(0, t)-\frac{\sqrt{2}}{3} \sigma d\left(3 b^{2}-2\right) K_{\alpha}(t)+P^{\prime}(0, t) .
$$

On the other hand, the junction conditions (121) gives

$$
\alpha_{(1)}^{(1)}=-\frac{\sqrt{2}}{6} \sigma d\left(K_{\gamma}(t)-\sqrt{2} b K_{\phi}(t)+f_{\gamma}(t)-\sqrt{2} f_{\phi}(t)\right)+P^{\prime}(0, t)-\frac{\kappa^{2}}{6}(\rho+U) d e^{\sqrt{2} b \hat{\phi}} .
$$

So we notice

$$
\begin{aligned}
\frac{\sqrt{2}}{3} \sigma d\left(1-6 b^{2}\right) f_{\alpha}(t)+g_{\alpha}(t)+\frac{\sqrt{2}}{6}\left(f_{\gamma}(t)\right. & \left.-\sqrt{2} b f_{\phi}(t)\right)+\frac{\kappa^{2}}{6}(\rho+U) d e^{\sqrt{2} b \hat{\phi}} \\
& =-\frac{\sqrt{2}}{6} \sigma d\left[-2\left(3 b^{2}-2\right) K_{\alpha}(t)+K_{\gamma}(t)-\sqrt{2} b K_{\phi}(t)\right]
\end{aligned}
$$

There appears the homogeneous solution in eq.(52), so we have to know the explicit form of it to obtain the effective Friedmann equation. In order to do so, we use the junction condition at $y=r$. Using it, we can write down the homogeneous solution in terms of the quantities on the negative 
tension brane. Indeed, projecting $\alpha_{(1)}^{\prime}$ onto $y=r$ we find

$$
\begin{aligned}
& \alpha_{(1)}^{\prime}(r, t)=\tilde{\alpha}_{(1)}^{(1)} \\
& =\left[\frac{\sqrt{2}}{3} \sigma d\left(1-6 b^{2}\right) f_{\alpha}(t)+g_{\alpha}(t)\right] \tilde{H}^{1-\left(1 / 3 b^{2}\right)}+\left[\frac{\sqrt{2}}{3} \sigma d\left(1-3 b^{2}\right) g_{\alpha}(t)+2 h_{\alpha}(t)\right] \tilde{H}^{-1 / 3 b^{2}} r \\
& \quad+\frac{\sqrt{2}}{3} \sigma d h_{\alpha}(t) \tilde{H}^{-1-\left(1 / 3 b^{2}\right)} r^{2}+k_{\alpha}^{\prime}(r, t)-\frac{\sqrt{2}}{3} \sigma d\left(3 b^{2}-2\right) K_{\alpha}(t) \tilde{H}^{-2 / 3 b^{2}}+P^{\prime}(r, t),
\end{aligned}
$$

and the junction condition (124) gives

$$
\begin{aligned}
\tilde{\alpha}_{(1)}^{(1)}=-\frac{\sqrt{2}}{6}[( & \left.K_{\gamma}(t)-\sqrt{2} b K_{\phi}(t)\right) \tilde{H}^{-2 / 3 b^{2}}+\left(f_{\gamma}(t)-\sqrt{2} b f_{\phi}(t)\right) \tilde{H}^{1-\left(1 / 3 b^{2}\right)} \\
& \left.+\left(g_{\gamma}(t)-\sqrt{2} b g_{\phi}(t)\right) \tilde{H}^{-1 / 3 b^{2}} r+\left(h_{\gamma}(t)-\sqrt{2} b h_{\phi}(t)\right) \tilde{H}^{-1-\left(1 / 3 b^{2}\right)} r^{2}\right] \\
& +P^{\prime}(r, t)+\frac{\kappa^{2}}{6}(\tilde{\rho}+\tilde{U}) d e^{\sqrt{2} b \hat{\phi}} .
\end{aligned}
$$

Combining them each other, we obtain

$$
\begin{aligned}
& {\left[\frac{\sqrt{2}}{3} \sigma d\left(1-6 b^{2}\right) f_{\alpha}(t)+g_{\alpha}(t)+\frac{\sqrt{2}}{6} \sigma d\left(f_{\gamma}(t)-\sqrt{2} b f_{\phi}(t)\right)\right] \tilde{H}^{1-\left(1 / 3 b^{2}\right)}} \\
& +\left[\frac{\sqrt{2}}{3} \sigma d\left(1-3 b^{2}\right) g_{\alpha}(t)+2 h_{\alpha}(t)+\frac{\sqrt{2}}{6} \sigma d\left(g_{\gamma}(t)-\sqrt{2} b g_{\phi}(t)\right)\right] \tilde{H}^{-1 / 3 b^{2}} r \\
& +\left[\frac{\sqrt{2}}{3} \sigma d h_{\alpha}(t)+\frac{\sqrt{2}}{6} \sigma d\left(h_{\gamma}(t)-\sqrt{2} b h_{\phi}(t)\right)\right] \tilde{H}^{-1-\left(1 / 3 b^{2}\right)} r^{2} \\
& +k_{\alpha}^{\prime}(r, t)-\frac{\kappa^{2}}{6}(\tilde{\rho}+\tilde{U}) d e^{\sqrt{2} b \hat{\phi}} \\
& =-\frac{\sqrt{2}}{6} \sigma d\left[-2\left(3 b^{2}-2\right) K_{\alpha}(t)+K_{\gamma}(t)-\sqrt{2} b K_{\phi}(t)\right] \tilde{H}^{-2 / 3 b^{2}}
\end{aligned}
$$

Explicit form of all homogeneous solutions are given in Appendix B. Now let us use the homogeneous solution (551). Substituting (55I) into (52), we finally obtain the effective Friedmann equation. The concrete form of the effective Friedmann equation (58) is given in the next section. Similarly, we can derive other effective equations. All of them are also given in the next section.

Here we note that one can not determine the homogeneous solution in one brane model because one knows the only one junction condition at the brane where one lives. 


\section{4D Effective equations}

\subsection{Friedmann equation, equations of motion for radion and scalar field}

Now we can explicitly write down four-dimensional effective equations. First, the evolution equations for scale factor are given by

$$
\begin{aligned}
& \ddot{\hat{\alpha}}+2 \dot{\hat{\alpha}}^{2}+\frac{1}{6} \dot{\hat{\phi}}^{2}=\frac{\sqrt{2}}{36} \kappa^{2} \sigma\left((1-3 w) \rho+4 U+3 \sqrt{2} b U^{\prime}\right) e^{-\sqrt{2} b \hat{\phi}}-\frac{1}{6} V\left(\phi^{(0)}\right), \\
& \ddot{\hat{\alpha}}+2 \dot{\hat{\alpha}}^{2}+\frac{1}{6} \dot{\hat{\phi}}^{2}=-\frac{\sqrt{2}}{36} \kappa^{2} \sigma\left((1-3 \tilde{w}) \tilde{\rho}+4 \tilde{U}+3 \sqrt{2} b \tilde{U}^{\prime}\right) e^{-\sqrt{2} b \phi} \tilde{H}^{-\frac{3 \Delta+4}{3 \Delta+8}} \\
& -\frac{1}{6} \tilde{H}^{\frac{4}{8+3 \Delta}} V\left(\tilde{\phi}^{(0)}\right)+\frac{2}{3 \Delta+8}(\ddot{d}+3 \dot{d} \dot{\hat{\alpha}}+\sqrt{2} b \dot{d} \dot{\hat{\phi}}) \tilde{H}^{-1}+\frac{3 \Delta+4}{(3 \Delta+8)^{2}} \dot{d}^{2} \tilde{H}^{-2}, \\
& \dot{\hat{\alpha}}^{2}+\sqrt{2} b \dot{\hat{\alpha}} \dot{\hat{\phi}}-\frac{1}{6} \dot{\hat{\phi}}^{2}=\frac{\sqrt{2}(\Delta+4)}{24(1-\tilde{H} \zeta)} \kappa^{2} \sigma\left[\rho+U+(\tilde{\rho}+\tilde{U}) \tilde{H}^{\frac{8}{3 \Delta+8}}\right] e^{-\sqrt{2} b \hat{\phi}} \\
& -\frac{\sqrt{2}}{24}(\Delta+4) \sigma d \frac{1}{1-\tilde{H} \zeta} \int_{0}^{r} d y H^{\frac{8}{8+3 \Delta}} V \\
& +\frac{3 \tilde{H}^{\zeta}(\Delta+4)}{1-\tilde{H}^{\zeta}}\left[-\frac{1}{3 \Delta+8} \dot{d} \dot{\hat{\alpha}} \tilde{H}^{-1}+\frac{1}{(3 \Delta+8)^{2}} \dot{d}^{2} \tilde{H}^{-2}\right] .
\end{aligned}
$$

The equation for $\hat{\phi}$ is given by

$$
\begin{gathered}
\ddot{\hat{\phi}}+3 \dot{\hat{\alpha}} \dot{\hat{\phi}}+\sqrt{2} b \dot{\hat{\phi}}^{2}=\frac{b \kappa^{2} \sigma}{6\left(1-\tilde{H}^{\zeta}\right)}\left[(1-3 w) \rho+4 U-\sqrt{2} b^{-1} U^{\prime}+\left((1-3 \tilde{w}) \tilde{\rho}+4 \tilde{U}-\sqrt{2} b^{-1} \tilde{U}^{\prime}\right) \tilde{H}^{\left.\frac{8}{3 \Delta+8}\right]}\right] e^{-\sqrt{2} b \hat{\phi}} \\
+\frac{\sqrt{2}}{8}(\Delta+4) \sigma d \frac{1}{1-\tilde{H}^{\zeta}} \int_{0}^{r} d y H^{\frac{8}{8+3 \Delta}}\left(V^{\prime}+\sqrt{2} b V\right) \\
\quad-\frac{1}{1-\tilde{H}^{\zeta}} \frac{\sqrt{2} b}{2} V\left(\phi^{(0)}\right)\left(1-\tilde{H}^{\frac{3 \Delta+16}{3 \Delta+8}} \frac{V\left(\tilde{\phi}^{(0)}\right)}{V\left(\phi^{(0)}\right)}\right) \\
+\frac{\tilde{H}^{\zeta}}{1-\tilde{H}^{\zeta}}\left[-\frac{6 \sqrt{2} b}{3 \Delta+8}(\ddot{d}+3 \dot{d} \dot{\hat{\alpha}}) \tilde{H}^{-1}-\frac{6 \Delta+20}{3 \Delta+8} \dot{d} \dot{\hat{\phi}}^{-1}+\frac{24 \sqrt{2} b}{(3 \Delta+8)^{2}} \dot{d}^{2} \tilde{H}^{-2}\right] . \quad \text { (59) }
\end{gathered}
$$

Finally the equation for $d(t)$ is given by

$$
\begin{aligned}
& \ddot{d}+3 \dot{d} \dot{\hat{\alpha}}+\sqrt{2} b \dot{d} \dot{\hat{\phi}}+\frac{3 \Delta+4}{2(3 \Delta+8)} \dot{d}^{2} \tilde{H}^{-1} \\
&=\frac{\sqrt{2}(3 \Delta+8)}{72} \tilde{H} \kappa^{2} \sigma\left[(1-3 w) \rho+4 U+3 \sqrt{2} b U^{\prime}+\left((1-3 \tilde{w}) \tilde{\rho}+4 \tilde{U}+3 \sqrt{2} b \tilde{U}^{\prime}\right) \tilde{H}^{-\frac{3 \Delta+4}{3 \Delta+8}}\right] e^{-\sqrt{2} b \hat{\phi}} \\
& \quad-\frac{8+3 \Delta}{12} \tilde{H} V\left(\phi^{(0)}\right)\left(1-\tilde{H}^{\frac{4}{8+3 \Delta}} \frac{V\left(\tilde{\phi}^{(0)}\right)}{V\left(\phi^{(0)}\right)}\right), \quad(60)
\end{aligned}
$$


where we defined

$$
\begin{aligned}
\tilde{H} & \equiv H(r, t)=1-d(t) \\
\zeta & \equiv \frac{3(\Delta+4)}{3 \Delta+8}
\end{aligned}
$$

To make the spacetime non-singular, we set the negative tension brane at $r=1 /\left(\sqrt{2} b^{2} \sigma\right)$ and assume $d<1$. We can also get the energy conservation law as follows,

$$
\begin{aligned}
\dot{\rho}+3(1+w) \rho \dot{\hat{\alpha}} & =0, \\
\dot{\tilde{\rho}}+3(1+\tilde{w}) \tilde{\rho}\left(\dot{\hat{\alpha}}-\frac{\sqrt{2}}{3 \Delta+8} \dot{d} \tilde{H}^{-1}\right) & =0,
\end{aligned}
$$

which can be derived from above equations. Because we have five equations for five unknown functions $(\hat{\alpha}, \hat{\phi}, d, \rho, \tilde{\rho})$ we can consistently solve these equations.

In the RS model, the effective equations are given by

$$
\begin{aligned}
\ddot{\hat{\alpha}}+2 \dot{\hat{\alpha}}^{2}+\frac{1}{6} \dot{\hat{\phi}}^{2} & =\frac{\kappa^{2} k}{6}((1-3 w) \rho+4 U)-\frac{V}{6}, \\
\ddot{\hat{\alpha}}+2 \dot{\hat{\alpha}}^{2}+\frac{1}{6} \dot{\hat{\phi}}^{2} & =-\frac{\kappa^{2} k}{6}((1-3 \tilde{w}) \tilde{\rho}+4 \tilde{U})+\ddot{d}+3 \dot{\hat{\alpha}} \dot{d}-\dot{d}^{2}-e^{-2 d} \frac{V}{6}, \\
\dot{\hat{\alpha}}^{2}-\frac{1}{6} \dot{\hat{\phi}}^{2} & =\frac{\kappa^{2} k}{3} \frac{e^{2 d}}{e^{2 d}-1}\left[\rho+U+(\tilde{\rho}+\tilde{U}) e^{-4 d}\right]+\frac{1}{12} V\left(1+e^{-2 d}\right) \\
\ddot{\hat{\phi}}+3 \dot{\hat{\alpha}} \dot{\hat{\phi}} & =-\frac{1}{e^{2 d}-1}\left(2 \dot{\hat{\alpha}} \dot{d}-\dot{d}^{2}\right), \\
\ddot{d}+3 \dot{\hat{\alpha}} \dot{d}-\dot{d}^{2} & =\frac{\kappa^{2} k}{6}\left[\left((1-3 w) \rho+4 U+((1-3 \tilde{w}) \tilde{\rho}+4 \tilde{U}) e^{-2 d}\right]-\frac{V}{6}\left(1-e^{-2 d}\right) .\right.
\end{aligned}
$$

Here we take $r=1 / k$.

\subsection{Effective Theory}

In the previous section, we derived effective four-dimensional equations. We try to interpret them in terms of the scalar-tensor gravity. In this section the effective theory for observers confined on a

positive tension brane is considered. In this section, we do not consider the potentials $U, \tilde{U}$ and $V$. 
The action for the scalar-tensor theory is given by

$$
S=\frac{1}{2 \kappa_{4}^{2}} \int d^{4} x \sqrt{-g}\left[\varphi R-\frac{\omega(\varphi)}{\varphi}(\partial \varphi)^{2}+\mathcal{L}_{m}\right]
$$

The Friedmann equation and the equation for $\varphi$ are given by

$$
\begin{aligned}
\dot{\hat{\alpha}}^{2}+\dot{\hat{\alpha}} \frac{\dot{\varphi}}{\varphi}-\frac{\omega(\varphi)}{6}\left(\frac{\dot{\varphi}}{\varphi}\right)^{2} & =\frac{\kappa_{4}^{2}}{3} \frac{\rho}{\varphi} \\
\ddot{\varphi}+\dot{\varphi}\left(3 \dot{\hat{\alpha}}+\frac{\dot{\omega}}{2 \omega+3}\right) & =\frac{\kappa_{4}^{2}}{2 \omega+3}(1-3 w) \rho .
\end{aligned}
$$

\subsubsection{Case1: $d=$ const.}

At first, let us consider the case where the radion is somehow stabilized, that is, $\dot{d}=0$. In such a case, we find that eq. (58) becomes

$$
\begin{aligned}
\dot{\hat{\alpha}}^{2}+\sqrt{2} b \dot{\hat{\alpha}} \dot{\hat{\phi}}-\frac{1}{6} \dot{\hat{\phi}}^{2} & =\frac{\sqrt{2}(\Delta+4)}{24\left(1-\tilde{H}^{\zeta}\right)} e^{-\sqrt{2} b \hat{\phi}}\left[\rho+\tilde{\rho} \tilde{H}^{\frac{8}{3 \Delta+8}}\right] \\
\ddot{\hat{\phi}}+3 \dot{\hat{\alpha}} \dot{\hat{\phi}}+\sqrt{2} b \dot{\hat{\phi}}^{2} & =\frac{b \kappa^{2} \sigma}{6\left(1-\tilde{H}^{\zeta}\right)}\left[(1-3 w) \rho+(1-3 \tilde{w}) \tilde{\rho} \tilde{H}^{\frac{8}{3 \Delta+8}}\right] e^{-\sqrt{2} b \hat{\phi}} .
\end{aligned}
$$

If we take

$$
\varphi \equiv e^{\sqrt{2} b \hat{\phi}}, \quad \omega=\frac{6}{3 \Delta+8}=\frac{1}{2 b^{2}},
$$

the equations become the same as (71). Then the effective theory can be identified with Brans-Dicke theory with Brans-Dicke parameter $\omega$ given in (74). We also find the four-dimensional gravitational constant becomes

$$
\kappa_{4}^{2} \equiv \frac{\sqrt{2}(\Delta+4)}{8\left(1-\tilde{H}^{\zeta}\right)} \kappa^{2} \sigma, \quad \tilde{\kappa}_{4} \equiv \kappa_{4}^{2} \tilde{H}^{\frac{8}{3 \Delta+8}}
$$

Here $\tilde{H}$ is constant because $d$ is constant now. It is interesting that the matter on the negative tension brane couples to the gravity with a different gravitational constant from the matter on a positive tension brane.

From the observational constraint, we need $\omega>3000$ at least at the late stage of cosmology [55]. So the dilaton coupling is strongly constraint as $b^{2}<1.6 \times 10^{-4}$. Thus in the HW theory with 
$b=1$, the stabilization mechanism for $\phi$ should be introduced in order to obtain an acceptable late time cosmology.

In RS model, the theory becomes Einstein theory and the four-dimensional gravitational constant is given by a well known formula;

$$
\kappa_{4}^{2}=\kappa^{2} k \frac{e^{2 d}}{e^{2 d}-1}, \quad \tilde{\kappa}_{4}^{2}=\kappa_{4}^{2} e^{-4 d} .
$$

\subsubsection{Case2: $\hat{\phi}=$ const.}

Next, we consider the case where the bulk scalar field is stabilized, i.e., $\dot{\hat{\phi}}=0$. This case has been studied by many authors in the context of the Randall-Sundrum model [50], [51], [56]. In such a case, eq.(58) becomes

$$
\begin{gathered}
\dot{\hat{\alpha}}^{2}-3 \frac{\tilde{H}^{\zeta}(\Delta+4)}{1-\tilde{H}^{\zeta}}\left[-\frac{1}{3 \Delta+8} \dot{\hat{\alpha}} \dot{d} \tilde{H}^{-1}+\frac{1}{(3 \Delta+8)^{2}} \dot{d}^{2} \tilde{H}^{-2}\right]=\frac{\sqrt{2}(\Delta+4)}{24\left(1-\tilde{H}^{\zeta}\right)} e^{-\sqrt{2} b \hat{\phi}}\left[\rho+\tilde{\rho} \tilde{H}^{\frac{8}{3 \Delta+8}}\right] \\
\ddot{d}+3 \dot{d} \dot{\hat{\alpha}}+\sqrt{2} b \dot{d} \dot{\hat{\phi}}+\frac{3 \Delta+4}{2(3 \Delta+8)} \dot{d}^{2} \tilde{H}^{-1}=\frac{\sqrt{2}(3 \Delta+8)}{72} \tilde{H} \kappa^{2} \sigma\left[(1-3 w) \rho+(1-3 \tilde{w}) \tilde{\rho} \tilde{H}^{-\frac{3 \Delta+4}{3 \Delta+8}}\right] e^{-\sqrt{2} b \hat{\phi}} .
\end{gathered}
$$

From these equations, if we take

$$
\varphi \equiv 1-\tilde{H}^{\zeta}, \quad \omega=\frac{2}{\Delta+4}\left(\frac{\varphi}{1-\varphi}\right)=\frac{3}{2\left(3 b^{2}+1\right)}\left(\frac{\varphi}{1-\varphi}\right)
$$

eqs.(177) and (178) can be written as (71). Now $\omega$ is a dynamical variable, so we conclude that scalar-tensor gravity is realized. The effective four-dimensional gravitational constant becomes

$$
\kappa_{4}^{2} \equiv \frac{\sqrt{2}(\Delta+4)}{8} \kappa^{2} \sigma, \quad \tilde{\kappa}_{4}^{2} \equiv \kappa_{4}^{2}(1-\varphi)^{\frac{8}{3(\Delta+4)}}=\kappa_{4}^{2}(1-\varphi)^{\frac{2}{3 b^{2}+1}}
$$

From the observational constraint $\omega>3000$, we find

$$
d>1-\left(\frac{1}{1500(\Delta+4)+1}\right)^{1 / \zeta} .
$$

The physical distance $D$ between the positive and negative tension brane is given by,

$$
D=\int_{0}^{\frac{1}{\sqrt{2} b^{2} \sigma}} d y d e^{\sqrt{2} b \hat{\phi}}=\frac{d}{\sqrt{2} b^{2} \sigma} e^{\sqrt{2} b \hat{\phi}} .
$$


Thus the constraint means the negative tension brane should be sufficiently far away from positive tension brane. For $b \neq 0$, the position of the singularity corresponds to $d=1$. Thus the constraint requires that the negative tension brane is close to the singularity.

In RS model with $\Delta=-8 / 3$, the BD field and BD parameter are given by

$$
\varphi \equiv 1-e^{-2 d}, \quad \omega=\frac{3}{2}\left(\frac{\varphi}{1-\varphi}\right)
$$

and the four dimensional gravitational constant is defined by

$$
\kappa_{4}^{2}=\kappa^{2} k, \quad \tilde{\kappa}_{4}^{2}=\kappa_{4}^{2}(1-\varphi)^{2}
$$

These results completely agree with the results obtained in [50, [51, [56]. The observational constraint implies

$$
D=d / k>4 / k
$$

\subsubsection{General Case}

In general cases, the effective theory becomes bi-scalar tensor theory. For small $b$ satisfying the constraint $b^{2}<1.6 \times 10^{-4}$, we can obtain a phenomenologically acceptable cosmology without introducing a stabilization mechanism if the negative tension brane is sufficiently away from the positive tension brane. However, the dynamics of the moduli fields strongly depends on the matter contents on both branes and the initial conditions for the moduli fields. Thus in many cases, it seems to be inevitable to introduce some stabilization mechanism for moduli fields. The stabilization problem of moduli fields are very general and serious problem which appears in all higher dimensional theories. Detailed analysis of the general dynamics of the moduli and investigation of the stabilization problem is beyond the scope of this paper. We will come back to this issue in future publications [57]. 


\section{Boundary inflation}

In this section, we consider a simple model for inflating branes driven by potentials $U, \tilde{U}$ and $V$. There are essentially two types of the inflation [4]. First, the potential energy of the brane potential $U$ can drive the inflation on our brane. This can be called "matter field inflation". Second, the potential energy in the bulk can also drive the inflation on our brane. This can be called "modular inflation". In general, one could have a mixture of both types of the inflation. In the following, we will provide a simple model for "matter field inflation" and "modular inflation" respectively. For simplicity, we do not consider the matter on both branes, that is $\rho=\tilde{\rho}=0$ through this section.

\subsection{Matter field inflation}

First, we consider a simple model for inflation driven by brane potential $U$. Then we assume that the bulk potential vanishes $V=0$. Furthermore, in order to avoid the negative tension brane hits the positive tension brane or singularity, we assume the moduli fields $d(t)$ and $\phi(t)$ are stabilized

by tuning potentials $U$ and $\tilde{U}$. From the equations of motion for $d(t)$ and $\phi(t)$, we notice that this can be achieved by choosing

$$
U=e^{2 \sqrt{2} b \phi} U_{0}, \quad \tilde{U}=-U \tilde{H}^{\frac{3 \Delta+4}{3 \Delta+8}},
$$

where $U_{0}$ is independent of $\phi$. Then it is possible to stabilize the moduli fields $d(t)$ and $\phi(t)$;

$$
d(t)=d_{*}=\text { const. } \quad \phi(t)=\phi_{*}=\text { const } .
$$

The effective equations become a simple equation

$$
\dot{\hat{\alpha}}^{2}=\frac{\sqrt{2}(\Delta+4)}{24} e^{\sqrt{2} b \phi_{*}} \kappa^{2} \sigma U_{0} .
$$

If one considers that the potential energy $U_{0}$ is provided by some matter field on the brane, the conventional four-dimensional inflationary scenario can be realized. We comment on the stability 
of the moduli fields $d(t)$ and $\phi(t)$. Let us consider small fluctuations of the moduli fields;

$$
d(t)=d_{*}+\delta d(t), \quad \phi(t)=\phi_{*}+\delta \phi(t)
$$

Linearizing the equations of motion for $\phi(\mathrm{t})$ and $d(t)$, we get the equations for fluctuations;

$$
\begin{array}{r}
\ddot{\delta d}+3 \dot{\hat{\alpha} \dot{\delta} d}+(3 \Delta+4) \dot{\hat{\alpha}}^{2} \delta d=0, \\
\ddot{\delta \phi}+3 \dot{\hat{\alpha} \dot{\delta} \phi}=-\frac{6 \sqrt{2}}{3 \Delta+8} \frac{\tilde{H}^{\zeta}}{1-\tilde{H} \zeta}(\ddot{\delta} d+3 \dot{\hat{\alpha} \dot{\delta} d}) .
\end{array}
$$

Then we found that for $3 \Delta+4>0$, the fluctuations acquire a positive mass squared, thus the solutions are stable. On the other hand for $3 \Delta+4<0$, the system of inflating two branes is unstable. This agrees with the result that in $\mathrm{RS}$ model with $3 \Delta+4=-4$, the inflating two branes are unstable and the radion fluctuation $\delta d(t)$ has a mass squared $-4 \dot{\hat{\alpha}}^{2}$. It would be interesting to quantify the effect of these moduli fluctuations on the inflationary scenario.

In RS model with no bulk scalar field, it is possible to construct a viable model without the stabilization of the moduli field $d(t)$. Let us consider the matter field inflation driven by potentials on a positive tension brane $U$. The effective equations are given by

$$
\begin{aligned}
\dot{\hat{\alpha}}^{2}+\frac{1}{e^{2 d}-1}\left(2 \dot{\hat{\alpha}} \dot{d}-\dot{d}^{2}\right) & =\frac{\kappa^{2} k}{3} \frac{e^{2 d}}{e^{2 d}-1} U, \\
\ddot{d}+3 \dot{\hat{\alpha}} \dot{d}-\dot{d}^{2} & =\frac{2 \kappa^{2} k}{3} U .
\end{aligned}
$$

The solution for $U=$ const. can be easily found as

$$
\dot{\hat{\alpha}}^{2}=\dot{d}^{2}=\frac{\kappa^{2} k}{3} U
$$

The fate of branes depend on initial conditions. If the initial condition for $d$ is properly chosen, the inflation on positive tension brane pushes the negative tension brane away. Thus the observational constraint on $d$ becomes easy to be satisfied. It is also interesting to consider a model where branes will collide, although there are many open questions in cosmology based on colliding branes. 


\subsection{Modular inflation}

Next let us consider an inflation driven by bulk potential $V$. In this case, the moduli $\phi$ acts as an inflaton. Thus we should not stabilize $\phi$. The stabilization mechanism should be switched on after inflation. In general, the other moduli $d(t)$ also participates in the dynamics of inflation. In order to simplify the model, we take a bulk potential as

$$
V=-\Lambda e^{-2 \sqrt{2} b \phi} \sigma^{2}
$$

Furthermore, as is done in the previous subsection, we stabilize $d(t)$ by tuning the brane potential $\tilde{U}$ on the negative tension brane as

$$
\tilde{U}=\frac{2 \sqrt{2} \sigma \Lambda}{\kappa^{2} \Delta}\left(\tilde{H}^{\frac{3 \Delta+4}{3 \Delta+8}}-\tilde{H}^{-1}\right) e^{-\sqrt{2} b \hat{\phi}}
$$

Then we can have $d(t)=d_{*}=$ const. and the effective equations become

$$
\begin{aligned}
\dot{\hat{\alpha}}^{2}+\sqrt{2} b \dot{\hat{\alpha}} \dot{\hat{\phi}}-\frac{1}{6} \dot{\hat{\phi}}^{2} & =-\frac{\Delta+4}{6 \Delta} \sigma \Lambda e^{-2 \sqrt{2} b \hat{\phi}} \\
\ddot{\hat{\phi}}+3 \dot{\hat{\alpha}} \dot{\hat{\phi}}+\sqrt{2} b \dot{\hat{\phi}}^{2} & =-\frac{2 \sqrt{2} b}{\Delta} \sigma \Lambda e^{-2 \sqrt{2} b \hat{\phi}} .
\end{aligned}
$$

This equations yield a power-law solutions for scale factor $e^{\hat{\alpha}}$ and scalar field $e^{\hat{\phi}}$. An interesting property of these solutions is that the power does not depend on the amplitude of the potential $\Lambda$ and it is determined only by the dilaton coupling $b$. We find that a power-law inflation can be realized for $-2>\Delta$.

Finally, let us consider a RS model. In this model, we can simply take $d \rightarrow \infty$ to neglect the contribution of the moduli $d(t)$ rather than stabilizing $d(t)$. Then the effective equations become

$$
\begin{aligned}
\dot{\hat{\alpha}}^{2} & =\frac{1}{6} \dot{\hat{\phi}}^{2}+\frac{1}{12} V, \\
\ddot{\hat{\phi}} & +3 \dot{\hat{\alpha}} \dot{\hat{\phi}}+\frac{1}{4} V^{\prime}=0 .
\end{aligned}
$$

If we define $\phi_{4}=\hat{\phi} / \kappa k^{1 / 2}$ and $V_{4}=V / 4 \kappa^{2} k$, these are completely the same as the equations obtained in conventional four-dimensional theory. Thus the bulk inflaton can mimic the four-dimensional inflaton dynamics [1]. 


\section{Conclusions and Future Works}

In this paper, we considered two branes model with bulk scalar field, which is a generalization of both Randall-Sundrum model and Hořava-Witten model. The bulk potential of the scalar field has a exponential functions $V_{b u l k}=\exp (-2 \sqrt{2} b \phi)$. We considered matter on both branes and arbitrary potentials in the bulk and on the brane. These matter and potentials induce the cosmological expansion of the brane as well as the time evolution of the bulk scalar field and radion. Because it is expected that the tension of the brane is much larger than the energy density of matter at late time cosmology, we have used the low-energy approximation to derive the equations which describe the cosmological evolution on the brane.

The main results of this paper are the four-dimensional effective equations (566)- (60) which govern the low energy dynamics of the two branes system. The important point is that once the four dimensional dynamics is determined by these effective equations, we can construct a correspondent full five-dimensional geometry. This correspondence would be useful to investigate the effect of the bulk geometry on four dimensional brane dynamics.

We also investigated observational constraints imposed on the model. We assume we are living on positive tension brane. First, we studied the case where the radion $d(t)$ is stabilized. In such a case, we can regard this model as Brans-Dicke theory with a Brans-Dicke parameter $1 / 2 b^{2}$. From the observational constraint, we concluded $b^{2}$ must be smaller than $1.6 \times 10^{-4}$. Second, we consider

the case where $\hat{\phi}=$ const. We found that this model can be identified with the scalar-tensor theory where the Brans-Dicke parameter is given by $\left(3 / 2\left(3 b^{2}+1\right)\right)(\varphi / 1-\varphi)$, where $\varphi$ is the BD field which is defined by $d(t)$. For RS model with $b=0$, this agrees with the result obtained in [50, [51, [56]. The observational constraint implies the negative tension brane should be sufficiently away from the positive tension brane. In general cases where there in no stabilization mechanism, the effective theory becomes bi-scalar tensor theory. 
Using the effective equations, we constructed several simple models for inflation. The inflation can be driven by potentials on the brane and bulk. We can construct various models for boundary inflation. It is very interesting to know we can distinguish these models from conventional four-dimensional model. The detailed analysis of these inflation models including the primordial fluctuations will be given in the near future.

\section{Note added}

After almost completing this work, we found [58]. In [58], the equations for the radion and the bulk scalar are derived using a moduli approximation and qualitatively the same result is obtained.

\section{Acknowledgements}

The work of K.K. was supported by JSPS.

\section{Appendix}

\section{A Five-dimensional equations}

\section{A.1 Einstein equations and scalar field equation}

Five-dimensional Einstein equation and scalar field equations are given by $(y, y):$

$$
\begin{aligned}
& \alpha^{\prime 2}+\alpha^{\prime} \beta^{\prime}-\left(\ddot{\alpha}+2 \dot{\alpha}^{2}-\dot{\alpha} \dot{\beta}\right) e^{2(\gamma-\beta)} \\
& =\frac{1}{6} \phi^{\prime 2}+\frac{1}{6} \dot{\phi}^{2} e^{2(\gamma-\beta)}-\frac{1}{6}\left[\left(b^{2}-\frac{2}{3}\right) e^{2(\gamma-\sqrt{2} b \phi)} \sigma^{2}-V(\phi) e^{2 \gamma}\right] .
\end{aligned}
$$


$(t, t):$

$$
\begin{aligned}
\alpha^{\prime \prime}+2 \alpha^{\prime 2}-\gamma^{\prime} \alpha^{\prime} & -\left(\dot{\alpha}^{2}+\dot{\gamma} \dot{\alpha}\right) e^{2(\gamma-\beta)} \\
& =-\frac{1}{6} \phi^{\prime 2}-\frac{1}{6} \dot{\phi}^{2} e^{2(\gamma-\beta)}-\frac{1}{6}\left[\left(b^{2}-\frac{2}{3}\right) e^{2(\gamma-\sqrt{2} b \phi)} \sigma^{2}-V(\phi) e^{2 \gamma}\right] \\
+ & \frac{1}{3} e^{\gamma}\left\{\left[-\sqrt{2} e^{-\sqrt{2} b \phi} \sigma-\kappa^{2}(\rho+U)\right] \delta(y)+\left[\sqrt{2} e^{-\sqrt{2} b \phi} \sigma-\kappa^{2}(\tilde{\rho}+\tilde{U})\right] \delta(y-r)\right\} .
\end{aligned}
$$

$(i, j):$

$$
\begin{aligned}
2 \alpha^{\prime \prime}-2 \gamma^{\prime} \alpha^{\prime}+2 & \alpha^{\prime} \beta^{\prime}+3 \alpha^{2}+\beta^{\prime \prime}+\beta^{\prime 2}-\beta^{\prime} \gamma^{\prime} \\
-(2 \ddot{\alpha}+ & \left.2 \dot{\gamma} \dot{\alpha}-2 \dot{\alpha} \dot{\beta}+3 \dot{\alpha}^{2}+\ddot{\gamma}+\dot{\gamma}^{2}-\dot{\beta} \dot{\gamma}\right) e^{2(\gamma-\beta)} \\
& =-\frac{1}{2} \phi^{2}+\frac{1}{2} \dot{\phi}^{2} e^{2(\gamma-\beta)}-\frac{1}{2}\left[\left(b^{2}-\frac{2}{3}\right) e^{2(\gamma-\sqrt{2} b \phi)} \sigma^{2}-V(\phi) e^{2 \gamma}\right] \\
& +e^{\gamma}\left\{\left[-\sqrt{2} e^{-\sqrt{2} b \phi} \sigma+\kappa^{2}(p-U)\right] \delta(y)+\left[\sqrt{2} e^{-\sqrt{2} b \phi} \sigma+\kappa^{2}(\tilde{p}-\tilde{U})\right] \delta(y-r)\right\} .
\end{aligned}
$$

$(y, t):$

$$
\dot{\alpha}^{\prime}-\dot{\gamma} \alpha^{\prime}-\dot{\alpha}\left(\beta^{\prime}-\alpha^{\prime}\right)=-\frac{1}{3} \dot{\phi} \phi^{\prime}
$$

Equation of motion for $\phi$ :

$$
\begin{aligned}
\phi^{\prime \prime}+3 \alpha^{\prime} \phi^{\prime}+\beta^{\prime} \phi^{\prime}-\gamma^{\prime} \phi^{\prime}-(\ddot{\phi}+3 \dot{\alpha} \dot{\phi}-\dot{\beta} \dot{\phi}+\dot{\gamma} \dot{\phi}) e^{2(\gamma-\beta)} & \\
=-\sqrt{2} b\left(b^{2}-\frac{2}{3}\right) e^{2(\gamma-\sqrt{2} b \phi)} \sigma^{2}+\frac{1}{2} \frac{d V}{d \phi} e^{2 \gamma} & \\
+e^{\gamma} & {\left[\left(-2 b e^{-\sqrt{2} b \phi} \sigma+\kappa^{2} \frac{d U}{d \phi}\right) \delta(y)+\left(2 b e^{-\sqrt{2} b \phi} \sigma+\kappa^{2} \frac{d \tilde{U}}{d \phi}\right) \delta(y-r)\right] . }
\end{aligned}
$$

We expand these equations in terms of $\varepsilon$.

\section{A.2 0th-oder}

The field equations of the 0 -th order are given as follows,

$$
\alpha_{(0)}^{\prime 2}+\alpha_{(0)}^{\prime} \beta_{(0)}^{\prime}=\frac{1}{6} \phi_{(0)}^{\prime 2}-\frac{1}{6}\left(b^{2}-\frac{2}{3}\right) \sigma^{2} e^{2\left(\gamma_{(0)}-\sqrt{2} b \phi_{(0)}\right)},
$$




$$
\begin{gathered}
\alpha_{(0)}^{\prime \prime}+2 \alpha_{(0)}^{\prime 2}-\gamma_{(0)}^{\prime} \alpha_{(0)}^{\prime}=-\frac{1}{6} \phi_{(0)}^{\prime 2}-\frac{1}{6}\left(b^{2}-\frac{2}{3}\right) \sigma^{2} e^{2\left(\gamma_{(0)}-\sqrt{2} b \phi_{(0)}\right)} \\
2 \alpha_{(0)}^{\prime \prime}-2 \gamma_{(0)}^{\prime} \alpha_{(0)}^{\prime}+2 \alpha_{(0)}^{\prime} \beta_{(0)}^{\prime}+3 \alpha_{(0)}^{\prime 2}+\beta_{(0)}^{\prime \prime}+\beta_{(0)}^{\prime 2}-\beta_{(0)}^{\prime} \gamma_{(0)}^{\prime} \\
=-\frac{1}{2} \phi_{(0)}^{\prime 2}-\frac{1}{2}\left(b^{2}-\frac{2}{3}\right) \sigma^{2} e^{2\left(\gamma_{(0)}-\sqrt{2} b \phi_{(0)}\right)}, \\
\dot{\alpha}_{(0)}^{\prime}-\dot{\gamma}_{(0)} \alpha_{(0)}^{\prime}-\dot{\alpha}_{(0)}\left(\beta_{(0)}^{\prime}-\alpha_{(0)}^{\prime}\right)=-\frac{1}{3} \dot{\phi}_{(0)} \phi_{(0)}^{\prime}, \\
\phi_{(0)}^{\prime \prime}+3 \alpha_{(0)}^{\prime} \phi_{(0)}^{\prime}+\beta_{(0)}^{\prime} \phi_{(0)}^{\prime}-\gamma_{(0)}^{\prime} \phi_{(0)}^{\prime}=-\sqrt{2} b\left(b^{2}-\frac{2}{3}\right) \sigma^{2} e^{2\left(\gamma_{(0)}-\sqrt{2} b \phi_{(0)}\right)} .
\end{gathered}
$$

Next, we can get the junction conditions at $y=0$ as follows,

$$
\begin{aligned}
& \alpha_{(0)}^{(1)}(t)=-\left.\frac{\sqrt{2}}{6} \sigma e^{\gamma_{(0)}-\sqrt{2} b \phi_{(0)}}\right|_{y=0}, \\
& \beta_{(0)}^{(1)}(t)=-\left.\frac{\sqrt{2}}{6} \sigma e^{\gamma_{(0)}-\sqrt{2} b \phi_{(0)}}\right|_{y=0}, \\
& \phi_{(0)}^{(1)}(t)=-\left.b \sigma e^{\gamma_{(0)}-\sqrt{2} b \phi_{(0)}}\right|_{y=0} .
\end{aligned}
$$

Similarly, we can get the junction conditions at $y=r$ as follows,

$$
\begin{aligned}
& \tilde{\alpha}_{(0)}^{(1)}(t)=-\left.\frac{\sqrt{2}}{6} \sigma e^{\gamma_{(0)}-\sqrt{2} b \phi_{(0)}}\right|_{y=r}, \\
& \tilde{\beta}_{(0)}^{(1)}(t)=-\left.\frac{\sqrt{2}}{6} \sigma e^{\gamma_{(0)}-\sqrt{2} b \phi_{(0)}}\right|_{y=r}, \\
& \tilde{\phi}_{(0)}^{(1)}(t)=-\left.b \sigma e^{\gamma_{(0)}-\sqrt{2} b \phi_{(0)}}\right|_{y=r} .
\end{aligned}
$$




\section{A.3 1st order}

In this subsection, we consider the equations of the order $\varepsilon^{1}$, which determine the behavior of $\hat{\alpha}(t), \hat{\phi}(t)$ and $d(t)$. They are given as,

$$
\begin{aligned}
& 2 \alpha_{(0)}^{\prime} \alpha_{(1)}^{\prime}+\alpha_{(0)}^{\prime} \beta_{(1)}^{\prime}+\alpha_{(1)}^{\prime} \beta_{(0)}^{\prime}-\left[\ddot{\alpha}_{(0)}+2 \dot{\alpha}_{(0)}^{2}-\dot{\alpha}_{(0)} \dot{\beta}_{(0)}\right] e^{2\left(\gamma_{(0)}-\beta_{(0)}\right)} \\
& =\frac{1}{3} \phi_{(0)}^{\prime} \phi_{(1)}^{\prime}+\frac{1}{6} \dot{\phi}_{(0)}^{2} e^{2\left(\gamma_{(0)}-\beta_{(0)}\right)}-\frac{1}{3}\left(b^{2}-\frac{2}{3}\right) \sigma^{2}\left(\gamma_{(1)}-\sqrt{2} b \phi_{(1)}\right) e^{2(\gamma(0)-\sqrt{2} b \phi(0))}+\frac{1}{6} e^{2 \gamma_{(0)}} V, \\
& \alpha_{(1)}^{\prime \prime}+4 \alpha_{(0)}^{\prime} \alpha_{(1)}^{\prime}-\gamma_{(0)}^{\prime} \alpha_{(1)}^{\prime}-\gamma_{(1)}^{\prime} \alpha_{(0)}^{\prime}-\left(\dot{\alpha}_{(0)}^{2}+\dot{\gamma}_{(0)} \dot{\alpha}_{(0)}\right) e^{2\left(\gamma_{(0)}-\beta_{(0)}\right)} \\
& =-\frac{1}{3} \phi_{(0)}^{\prime} \phi_{(1)}^{\prime}-\frac{1}{6} \dot{\phi}_{(0)}^{2} e^{2\left(\gamma_{(0)}-\beta_{(0)}\right)}-\frac{1}{3}\left(b^{2}-\frac{2}{3}\right) \sigma^{2}\left(\gamma_{(1)}-\sqrt{2} b \phi_{(1)}\right) e^{2\left(\gamma_{(0)}-\sqrt{2} b \phi_{(0)}\right)}+\frac{1}{6} e^{2 \gamma_{(0)}} V, \\
& 2 \alpha_{(1)}^{\prime \prime}-2 \gamma_{(0)}^{\prime} \alpha_{(1)}^{\prime}-2 \gamma_{(1)}^{\prime} \alpha_{(0)}^{\prime}+2 \alpha_{(0)}^{\prime} \beta_{(1)}^{\prime}+2 \alpha_{(1)}^{\prime} \beta_{(0)}^{\prime}+6 \alpha_{(0)}^{\prime} \alpha_{(1)}^{\prime}+\beta_{(1)}^{\prime \prime}+2 \beta_{(0)}^{\prime} \beta_{(1)}^{\prime}-\beta_{(0)}^{\prime} \gamma_{(1)}^{\prime}-\beta_{(1)}^{\prime} \gamma_{(0)}^{\prime} \\
& +\left(-2 \ddot{\alpha}_{(0)}-2 \dot{\gamma}_{(0)} \dot{\alpha}_{(0)}+2 \dot{\alpha}_{(0)} \dot{\beta}_{(0)}-3 \dot{\alpha}_{(0)}^{2}-\ddot{\gamma}_{(0)}-\dot{\gamma}_{(0)}^{2}+\dot{\beta}_{(0)} \dot{\gamma}_{(0)}\right) e^{2\left(\gamma_{(0)}-\beta_{(0)}\right)} \\
& =-\phi_{(0)}^{\prime} \phi_{(1)}^{\prime}+\frac{1}{2} \dot{\phi}_{(0)}^{2} e^{2\left(\gamma_{(0)}-\beta_{(0)}\right)}-\left(b^{2}-\frac{2}{3}\right) \sigma^{2}\left(\gamma_{(1)}-\sqrt{2} b \phi_{(1)}\right) e^{2\left(\gamma_{(0)}-\sqrt{2} b \phi_{(0)}\right)}+\frac{1}{2} e^{2 \gamma_{(0)}} V, \\
& \dot{\alpha}_{(1)}^{\prime}-\left(\dot{\gamma}_{(0)} \alpha_{(1)}^{\prime}+\dot{\gamma}_{(1)} \alpha_{(0)}^{\prime}\right) \\
& -\left[\dot{\alpha}_{(0)}\left(\beta_{(1)}^{\prime}-\alpha_{(1)}^{\prime}\right)+\dot{\alpha}_{(1)}\left(\beta_{(0)}^{\prime}-\alpha_{(0)}^{\prime}\right)\right]=-\frac{1}{3}\left(\dot{\phi}_{(0)} \phi_{(1)}^{\prime}+\dot{\phi}_{(1)} \phi_{(0)}^{\prime}\right), \\
& \phi_{(1)}^{\prime \prime}+3 \alpha_{(0)}^{\prime} \phi_{(1)}+3 \alpha_{(1)}^{\prime} \phi_{(0)}^{\prime}+\beta_{(0)}^{\prime} \phi_{(1)}^{\prime}+\beta_{(1)}^{\prime} \phi_{(0)}^{\prime}-\gamma_{(0)}^{\prime} \phi_{(1)}^{\prime}-\gamma_{(1)}^{\prime} \phi_{(0)}^{\prime} \\
& -\left(\ddot{\phi}_{(0)}+3 \dot{\alpha}_{(0)} \dot{\phi}_{(0)}-\dot{\beta}_{(0)} \dot{\phi}_{(0)}+\dot{\gamma}_{(0)} \dot{\phi}_{(0)}\right) e^{2\left(\gamma(0)-\beta_{(0)}\right)} \\
& =-2 \sqrt{2} b\left(b^{2}-\frac{2}{3}\right) \sigma^{2}\left(\gamma_{(1)}-\sqrt{2} b \phi_{(1)}\right) e^{2\left(\gamma_{(0)}-\sqrt{2} b \phi_{(0)}\right)}+\frac{1}{2} \frac{d V}{d \phi} .
\end{aligned}
$$


Similarly, we can derive junction conditions at $y=0$ and $y=r$ as follows,

$$
\begin{aligned}
& \alpha_{(1)}^{(1)}(t)=-\frac{\sqrt{2}}{6}\left(\gamma_{(1)}-\sqrt{2} b \phi_{(1)}\right) \sigma e^{\gamma_{(0)}-\sqrt{2} b \phi_{(0)}}-\left.\frac{\kappa^{2}}{6}(\rho+U) e^{\gamma_{(0)}}\right|_{y=0}, \\
& \beta_{(1)}^{(1)}(t)=-\frac{\sqrt{2}}{6}\left(\gamma_{(1)}-\sqrt{2} b \phi_{(1)}\right) \sigma e^{\gamma_{(0)}-\sqrt{2} b \phi_{(0)}}+\left.\frac{\kappa^{2}}{6}(3 p+2 \rho-U) e^{\gamma_{(0)}}\right|_{y=0}, \\
& \phi_{(1)}^{(1)}(t)=-b\left(\gamma_{(1)}-\sqrt{2} b \phi_{(1)}\right) \sigma e^{\gamma_{(0)}-\sqrt{2} b \phi_{(0)}}+\left.\frac{\kappa^{2}}{2} \frac{d U}{d \phi} e^{\gamma_{(0)}}\right|_{y=0}, \\
& \tilde{\alpha}_{(1)}^{(1)}(t)=-\frac{\sqrt{2}}{6}\left(\gamma_{(1)}-\sqrt{2} b \phi_{(1)}\right) \sigma e^{\gamma_{(0)}-\sqrt{2} b \phi_{(0)}}+\left.\frac{\kappa^{2}}{6}(\tilde{\rho}+\tilde{U}) e^{\gamma_{(0)}}\right|_{y=r}, \\
& \tilde{\beta}_{(1)}^{(1)}(t)=-\frac{\sqrt{2}}{6}\left(\gamma_{(1)}-\sqrt{2} b \phi_{(1)}\right) \sigma e^{\gamma_{(0)}-\sqrt{2} b \phi_{(0)}}-\left.\frac{\kappa^{2}}{6}(3 \tilde{p}+2 \tilde{\rho}-\tilde{U}) e^{\gamma_{(0)}}\right|_{y=r}, \\
& \tilde{\phi}_{(1)}^{(1)}(t)=-b\left(\gamma_{(1)}-\sqrt{2} b \phi_{(1)}\right) \sigma e^{\gamma_{(0)}-\sqrt{2} b \phi_{(0)}}-\left.\frac{\kappa^{2}}{2} \frac{d \tilde{U}}{d \phi} e^{\gamma_{(0)}}\right|_{y=r} .
\end{aligned}
$$




\section{B Solutions for 1st Order Equations}

\section{B.1 Homogeneous solutions}

Here we will write down the homogeneous solutions in terms of $\tilde{\rho}, \tilde{U}$.

$$
\begin{aligned}
& K_{\alpha}=-\frac{\sqrt{2} \kappa^{2} \sigma^{-1}}{3 \Delta+8}(\tilde{\rho}+\tilde{U}) e^{\sqrt{2} b \hat{\phi}} \\
& +\frac{2 \sqrt{2} \sigma^{-2}}{\Delta} e^{2 \sqrt{2} b \hat{\phi}}\left[-\frac{2 \sqrt{2}}{(\Delta+4)}\left(\dot{\hat{\alpha}}^{2}+\sqrt{2} b \dot{\hat{\alpha}} \dot{\hat{\phi}}-\frac{1}{6} \dot{\hat{\phi}}^{2}\right) \tilde{H}^{\frac{3(\Delta+4)}{3 \Delta+8}}\right. \\
& \left.+\frac{6 \sqrt{2}}{3 \Delta+8} \dot{d} \dot{\hat{\alpha}} \tilde{H}^{\frac{4}{3 \Delta+8}}+\frac{6 \sqrt{2}}{(3 \Delta+8)^{2}} \dot{d}^{2} \tilde{H}^{-\frac{3 \Delta+4}{3 \Delta+8}}\right] \\
& -\frac{\sqrt{2} \sigma^{-1} d}{3 \Delta} e^{2 \sqrt{2} b \hat{\phi}} \tilde{H}^{-\frac{8}{3 \Delta+8}} \int_{0}^{r} d y H^{\frac{8}{3 \Delta+8}} V \\
& K_{\beta}=\frac{\sqrt{2} \kappa^{2} \sigma^{-1}}{3 \Delta+8}((3 \tilde{w}+2) \tilde{\rho}-\tilde{U}) e^{\sqrt{2} b \hat{\phi}} \\
& +\frac{2 \sqrt{2} \sigma^{-2}}{\Delta} e^{2 \sqrt{2} b \hat{\phi}}\left[-\frac{2 \sqrt{2}}{(\Delta+4)}\left(2 \ddot{\hat{\alpha}}+\dot{\hat{\alpha}}^{2}+\sqrt{2} b \ddot{\hat{\phi}}+\frac{3 \Delta+13}{6} \dot{\hat{\phi}}^{2}\right) \tilde{H}^{\frac{3(\Delta+4)}{3 \Delta+8}}\right. \\
& \left.+\frac{6 \sqrt{2}}{3 \Delta+8}\left(\frac{\ddot{d}}{d}+2 \sqrt{2} b \frac{\dot{d}}{\dot{\phi}}\right) \tilde{H}^{\frac{4}{3 \Delta+8}}-\frac{6 \sqrt{2}}{(3 \Delta+8)^{2}} \dot{d}^{2} \tilde{H}^{-\frac{3 \Delta+4}{3 \Delta+8}}\right] \\
& -\frac{\sqrt{2} \sigma^{-1} d}{\Delta} e^{2 \sqrt{2} b \hat{\phi}} \tilde{H}^{-\frac{8}{3 \Delta+8}} \int_{0}^{r} d y H^{\frac{8}{3 \Delta+8}}\left[\sqrt{2} b V^{\prime}+\left(1+2 b^{2}\right) V\right]-\frac{\sigma^{-2}}{\Delta} e^{2 \sqrt{2} b \phi} H V, \\
& K_{\phi}=\frac{\sqrt{2} \kappa^{2} \sigma^{-1}}{\Delta} U^{\prime} e^{\sqrt{2} b \hat{\phi}} \\
& +\frac{2 \sqrt{2} \sigma^{-2}}{\Delta} e^{2 \sqrt{2} b \hat{\phi}}\left[-\frac{2 \sqrt{2}}{(\Delta+4)}\left(\ddot{\hat{\phi}}+3 \dot{\hat{\alpha}} \dot{\hat{\phi}}+\sqrt{2} b \dot{\hat{\phi}}^{2}-3 \sqrt{2} b\left(\ddot{\hat{\alpha}}+2 \dot{\hat{\alpha}}^{2}+\frac{1}{6} \dot{\hat{\phi}}^{2}\right)\right)\right. \\
& \left.+\frac{6 \sqrt{2}}{3 \Delta+8} \dot{d} \dot{\hat{\phi}} \tilde{H} \frac{4}{3 \Delta+8}-\frac{36 b}{(3 \Delta+8)^{2}} \dot{d}^{2} \tilde{H}^{-\frac{3 \Delta+4}{3 \Delta+8}}\right] \\
& -\frac{\sqrt{2} \sigma^{-1} d}{\Delta} e^{2 \sqrt{2} b \hat{\phi}} \tilde{H}^{-\frac{8}{3 \Delta+8}} \int_{0}^{r} d y H^{\frac{8}{3 \Delta+8}}(V+\sqrt{2} b V) .
\end{aligned}
$$




\section{B.2 Particular solutions}

The particular solutions for 1 st order equations are given in eqs.(43)-(46). The concrete form of them becomes as follows;

$$
\begin{aligned}
& f_{\alpha}=\frac{4 \sigma^{-2}}{(\Delta+4)(\Delta+2)} e^{2 \sqrt{2} b \hat{\phi}}\left[\dot{\hat{\alpha}}^{2}+\sqrt{2} b \dot{\hat{\alpha}} \dot{\hat{\phi}}-\frac{1}{6} \dot{\hat{\phi}}^{2}-\frac{3(\Delta+4)}{3 \Delta+4}\left(\frac{\dot{d}}{d} \dot{\hat{\alpha}}+\frac{1}{2} \frac{\dot{d}^{2}}{d^{2}}\right)\right], \\
& f_{\beta}=\frac{4 \sigma^{-2}}{(\Delta+4)(\Delta+2)} e^{2 \sqrt{2} b \hat{\phi}}\left[2 \ddot{\hat{\alpha}}+\dot{\hat{\alpha}}^{2}+\sqrt{2} b \ddot{\hat{\phi}}+\frac{3 \Delta+13}{6} \dot{\hat{\phi}}^{2}\right. \\
& \left.-\frac{3(\Delta+4)}{3 \Delta+4}\left(2 \sqrt{2} b \frac{\dot{d}}{d} \dot{\hat{\phi}}+\frac{\ddot{d}}{d}-\frac{1}{2} \frac{\dot{d}^{2}}{d^{2}}\right)\right] \\
& f_{\phi}=\frac{4 \sigma^{-2}}{(\Delta+4)(\Delta+2)} e^{2 \sqrt{2} b \hat{\phi}}\left[\ddot{\hat{\phi}}+3 \dot{\hat{\alpha}} \dot{\hat{\phi}}+\sqrt{2} b \dot{\hat{\phi}}^{2}-3 \sqrt{2} b\left(\ddot{\hat{\alpha}}+2 \dot{\hat{\alpha}}^{2}+\frac{1}{6} \dot{\hat{\phi}}^{2}\right)\right. \\
& \left.-\frac{3(\Delta+4)}{3 \Delta+4}\left(\frac{\dot{d}}{d} \dot{\hat{\phi}}+\frac{3 \sqrt{2}}{2} b \frac{\dot{d}^{2}}{d^{2}}\right)\right] \\
& f_{\gamma}=\sqrt{2} b f_{\phi} \\
& g_{\alpha}=-\frac{3 \sqrt{2} \sigma^{-1}}{3 \Delta+4} e^{2 \sqrt{2} b \hat{\phi}}\left(2 \dot{d} \dot{\hat{\alpha}}+\frac{\dot{d}^{2}}{d}\right) \text {, } \\
& g_{\beta}=-\frac{3 \sqrt{2} \sigma^{-1}}{3 \Delta+4} e^{2 \sqrt{2} b \hat{\phi}}\left(2 \ddot{d}+4 \sqrt{2} b \dot{d} \dot{\hat{\phi}}-\frac{\dot{d}^{2}}{d}\right) \text {, } \\
& g_{\phi}=-\frac{18 \sigma^{-1}}{3 \Delta+4} e^{2 \sqrt{2} b \hat{\phi}}\left(b \frac{\dot{d}^{2}}{d}+\frac{\sqrt{2}}{3} \dot{\dot{d}} \dot{\hat{\phi}}\right) \text {, } \\
& g_{\gamma}=\sqrt{2} b g_{\phi}, \\
& h_{\alpha}=-\frac{1}{4} \dot{d}^{2} e^{2 \sqrt{2} b \hat{\phi}}, \\
& h_{\beta}=\frac{1}{4} \dot{d}^{2} e^{2 \sqrt{2} b \hat{\phi}}, \\
& h_{\phi}=-\frac{3 \sqrt{2}}{4} b \dot{d}^{2} e^{2 \sqrt{2} b \hat{\phi}}, \\
& h_{\gamma}=\sqrt{2} b h_{\phi} \text {. }
\end{aligned}
$$

For $\Delta=-2$ and/or $\Delta=-4 / 3$, the particular solutions should be treated separately. But even in such cases, we get the same effective $4 \mathrm{D}$ equations as we have shown in Sec.4. 


\section{Homogeneous and Particular Solutions in RS model}

\section{C.1 Homogeneous solutions}

$$
\begin{aligned}
& \alpha_{(1)}(y, t)=K_{\alpha}(t) e^{4 k d y}+F_{\alpha}(t)+P(y, t), \\
& \beta_{(1)}(y, t)=K_{\beta}(t) e^{4 k d y}+F_{\beta}(t)+P(y, t), \\
& \gamma_{(1)}(y, t)=K_{\gamma}(t) e^{4 k d y}-\frac{3 \sqrt{2}}{d} P^{\prime}(y, t), \\
& \phi_{(1)}(y, t)=K_{\phi}(t) e^{4 k d y}+F_{\phi}(t),
\end{aligned}
$$

where $F_{\alpha}, F_{\beta}, F_{\phi}$ and $P$ are arbitrary functions which do not affect the resultant $4 \mathrm{D}$ equations. $K_{\alpha}, K_{\beta}, K_{\gamma}$ and $K_{\phi}$ yield the the constraints;

$$
\begin{gathered}
3 K_{\alpha}+K_{\beta}+K_{\gamma}=0 \\
\dot{K}_{\alpha}+\left(K_{\alpha}-K_{\beta}\right) \dot{\hat{\alpha}}+\frac{1}{3} K_{\phi} \dot{\hat{\phi}}+\frac{1}{4} \dot{K}_{\gamma}=0 .
\end{gathered}
$$

Here we define $k=(\sqrt{2} / 6) \sigma$ as in Sec.2.3.

\section{C.2 Particular solutions}

The particular solutions are given by

$$
\begin{aligned}
& \alpha_{(1)}=\left[-\frac{1}{4 k^{2}}\left(\dot{\hat{\alpha}}^{2}-\frac{1}{6} \dot{\hat{\phi}}+\frac{\dot{d}}{\dot{d}} \dot{\hat{\alpha}}+\frac{1}{2} \frac{\dot{d}^{2}}{d^{2}}\right)+\frac{1}{2 k}\left(\dot{d} \dot{\hat{\alpha}}+\frac{1}{2} \frac{\dot{d}^{2}}{d^{2}}\right) y-\frac{1}{4} \dot{d}^{2} y^{2}\right] e^{2 k d y}, \\
& \beta_{(1)}=\left[-\frac{1}{4 k^{2}}\left(2 \ddot{\hat{\alpha}}+\dot{\hat{\alpha}}^{2}+\frac{5}{6} \dot{\hat{\phi}}+\frac{\ddot{d}}{d}-\frac{1}{2} \frac{\dot{d}^{2}}{d^{2}}\right)+\frac{1}{2 k}\left(\ddot{d}-\frac{1}{2} \frac{\dot{d}^{2}}{d^{2}}\right) y+\frac{1}{4} \dot{d}^{2} y^{2}\right] e^{2 k d y}, \\
& \phi_{(1)}=\left[-\frac{1}{4 k^{2}}\left(\ddot{\hat{\phi}}+3 \dot{\hat{\alpha}} \dot{\hat{\phi}}+\frac{\dot{d}}{\dot{\phi}}\right)+\frac{1}{2 k} \dot{\phi} \dot{\hat{\phi}}\right] e^{2 k d y}, \\
& \gamma_{(1)}=0 .
\end{aligned}
$$




\section{References}

[1] P. Hořava and E. Witten, Nucl. Phys. B460 (1996) 506.

[2] P. Hořava and E. Witten, Nucl. Phys. B475 (1996) 94.

[3] E. Witten, Nucl. Phys. B471 (1996) 135.

[4] A. Lukas, B. A. Ovrut, K. S. Stelle and D. Waldram, Nucl. Phys. B552 (1999) 246.

[5] A. Lukas, B. A. Ovrut, K. S. Stelle and D. Waldram, Phys. Rev. D59 (1999) 086001.

[6] A. Lukas, B. A. Ovrut, K. S. Stelle and D. Waldram, Phys. Rev. D60 (1999) 086001.

[7] U. Ellwanger, hep-th/0001126.

[8] H. S. Reall, Phys. Rev. D59 (1999) 103506.

[9] S. Kobayashi, K. Koyama and J. Soda, Phys. Lett. B501 (2001) 157-164.

[10] Y. Himemoto and M. Sasaki, Phys. Rev. D63 (2001) 044015.

[11] Y. Himemoto, T. Tanaka and M. Sasaki, Phys. Rev. D65 (2002) 104020.

[12] N. Sago, Y. Himemoto and M. Sasaki, Phys. Rev. D65 (2002) 024014.

[13] K. Uzawa and J. Soda, Mod. Phys. Lett. A16 (2001) 1089.

[14] N. Arkani-Hamed, S. Dimopoulos, N. Kaloper and R. Sundrum, Phys. Lett.B480 (2000) 193.

[15] S. Kachru, M. Schulz and E. Silverstein, Phys. Rev. D62 (2000) 045021.

[16] C. Csaki, J. Erlich, C. Grojean and T. Hollowood, Nucl. Phys. B584 (2000) 359.

[17] P. Binetruy, J. M. Cline and C. Grojean, Phys. Lett. B489 (2000) 403.

[18] S. Kobayashi, K. Koyama and J. Soda, Phys. Rev D65 (2002) 064014. 
[19] O. DeWolfe, D. Z. Freedman, S. S. Gubser and A. Karch, Phys. Rev. D62 (2000) 046008.

[20] M. Gremm, Phys. Lett. B478 (2000) 434.

[21] C. Csáki, J. Erlich, T. J. Hollowood and Y. Shirman, Nucl. Phys. B581 (2000) 309.

[22] L. Randall and R. Sundrum, Phys. Rev. Lett. 83. (1999) 3370.

[23] L. Randall and R. Sundrum, Phys. Rev. Lett. 83. (1999) 4690.

[24] D. Langlois and M Rodríguez-Martínez, Phys. Rev. D64 (2001) 123507.

[25] A. Mennim and R. A. Battye, Class. Quant. Grav. 18 (2001) 2171.

[26] W. D. Goldberger and M. B. Wise, Phys. Rev. Lett. 83 (1999) 4922.

[27] W. D. Goldberger and M. B. Wise, Phys. Lett. B475 (2000) 275.

[28] C. Csáki, M. L. Graesser and G. D. Kribs, Phys. Rev. D63 (2001) 065002.

[29] T. Tanaka and X. Montes, Nucl. Phys. B582 (2000) 259.

[30] H. Kudoh and T. Tanaka, Phys. rev. D65 (2002) 104034.

[31] H. Kudoh and T. Tanaka, hep-th/0205041.

[32] J. M. Cline, J. Vinet, JHEP 0202 (2002) 042.

[33] J. M. Cline, H. Firouzjahi, Phys. Lett. B495 (2000) 271-276.

[34] C. Charmousis, R. Gregory and V. A. Rubakov, Phys. Rev. D62 (2000) 067505.

[35] C. Csáki, M. L. Graesser, L. Randall and J. Terning, Phys. Rev. D62 (2000) 045015.

[36] Z. Chacko and P. J. Fox, Phys. Rev. D64 (2001) 024015.

[37] U. Gen and M. Sasaki, Prog. Theor. Phys. 105 (2001) 591; gr-qc/0201031

[38] P. Binetruy, C Deffayet, D Langlois, Nucl. Phys. B615, 219, (2001). 
[39] T. Wiseman, Class. Quant. Grav. 19, 3083, 2002.

[40] K. Koyama, gr-qc/0204047, Phys.Rev. D in press.

[41] T. Chiba, Phys. Rev D62, 021502, (2000);

[42] D. Longlois and L. Sorbo, Phys. Lett. B543 (2002) 155.

[43] A. Das and A. Mitov, Phys. rev. D66 (2002) 045030.

[44] Ph. Brax, C. van de Bruck, A. C. Davis and C. S. Rhodes, hep-th/0201191.

[45] R. Kallosh, L. Kofman, A. Linde and A. Tseytlin, Phys. Rev. D64 (2001) 123524.

[46] G. W. Gibbons and P. K. Townsend, Nucl. Phys. B282 (1987) 610.

[47] A. A. Tseytlin, Phys. Lett. B334 (1994) 315.

[48] S. Mukohyama, Phys. Rev. D 65, (2002), 124025

[49] A. Lukas, B. A. Ovrut and D. Waldram, Phys. Rev. D61 (1999) 023506.

[50] S. Kanno and J. Soda, Phys. Rev D66 (2002) 043526.

[51] S. Kanno and J. Soda, hep-th/0207029.

[52] M. Cvetič, Phys. Lett. B341, (1994) 160.

[53] D. Youm, Nucl. Phys. B576 (2000) 106.

[54] M. Cvetič, H. Lü and N. Pope, Phys. Rev. D63 (2001) 086004.

[55] C. M. Will, Living Rev. Rel. 4 (2001) 4.

[56] J. Garriga and T. Tanaka, Phys. Rev. Lett. 84 (2000) 2778.

[57] S. Kobayashi and K. Koyama, work in progress.

[58] Ph. Brax, C. van de Bruck, A.-C. Davis and C. S. Rhodes, hep-th/0209158. 